\title{
Thermomechanical Characterisation of Copper Diamond and Benchmarking with the MultiMat Experiment
}

\author{
Marcus Portelli (D), ${ }^{1,2}$ Michele Pasquali (D), ${ }^{3}$ Federico Carra ${ }^{(D)},{ }^{2}$ Alessandro Bertarelli (D), \\ Pierluigi Mollicone $\mathbb{D D}^{1}$, Nicholas Sammut $\mathbb{D},{ }^{1}$ Óscar Sacristán de Frutos $\mathbb{D}^{\mathrm{D}}{ }^{2}$ \\ Jorge Guardia Valenzuela $\mathbb{D}^{2},{ }^{2}$ Erich Neubauer $\left(\mathbb{D},{ }^{4}\right.$ Michael Kitzmantel $\left(\mathbb{D},{ }^{4}\right.$ \\ and David Grech (D) $^{4}$ \\ ${ }^{1}$ University of Malta, Msida 2080, Malta \\ ${ }^{2}$ CERN, Esplanade des Particules 1, 1211, Geneva 23, Switzerland \\ ${ }^{3}$ Sapienza University of Rome, Via Eudossiana 18, Rome 00184, Italy \\ ${ }^{4}$ RHP-Technology GmbH, Seibersdorf 2444, Austria
}

Correspondence should be addressed to Marcus Portelli; marcusportelli@outlook.com

Received 13 July 2020; Revised 25 November 2020; Accepted 12 December 2020; Published 5 January 2021

Academic Editor: Trupti Ranjan Lenka

Copyright (C) 2021 Marcus Portelli et al. This is an open access article distributed under the Creative Commons Attribution License, which permits unrestricted use, distribution, and reproduction in any medium, provided the original work is properly cited.

\begin{abstract}
The High-Luminosity Large Hadron Collider upgrade at CERN will result in an increase in the energy stored in the circulating particle beams, making it necessary to assess the thermomechanical performance of currently used and newly developed materials for use in beam intercepting devices such as collimators and absorbers. This study describes the thermomechanical characterisation of a novel copper diamond grade selected for use in tertiary collimators of the HL-LHC. The data obtained are used to build an elastoplastic material model and implemented in numerical simulations performed to benchmark experimental data obtained from the recently completed MultiMat experiment conducted at CERN's HiRadMat facility, where various materials shaped as slender rods were tested under particle beam impact. The analyses focus on the dynamic longitudinal and flexural response of the material, with results showing that the material model is capable of replicating the material behaviour to a satisfactory level in both thermal and structural domains, accurately matching experimental measurements in terms of temperature, frequency content, and amplitude.
\end{abstract}

\section{Introduction}

This work presents the material characterisation of the copper diamond (CuCD) RHP3434 grade conducted at CERN's facilities, which includes a combination of temperature-dependent measurements for density, specific heat capacity, conductivity, and coefficient of thermal expansion, along with impulse excitation technique (IET) tests for the derivation of the dynamic elastic properties and a four-point bending test for the stressstrain behaviour of the material in the plastic regime. The material model derived from the characterisation was implemented in transient thermomechanical simulations performed in the finite element analysis software ANSYS, modelling various types of particle beam impacts on CuCD samples tested at CERN.

The study is organised as follows. Following the introduction, Section 2 details information about copper diamond and the grade studied. Section 3 details the material characterisation campaign carried out on the CuCD RHP3434 grade, consisting of measurements of temperature-dependent properties, elastic constants, and the plasticity curve. Section 4 presents the benchmarking of the material model by implementing it in a simulation compared with data obtained in the MultiMat experiment. This is followed by a discussion of results and future analyses were proposed for an extensive modelling of the material behaviour in a wide variety of applications and scenarios. 


\section{Copper Diamond}

Copper diamond is a novel composite material developed as a baseline material for use in collimators in the upcoming HL-LHC upgrade of the CERN's LHC particle accelerator, specifically as a replacement of the tungsten heavy alloy Inermet180. The material grade tested in this study, developed by RHP Technology [1], consists of diamond particles dispersed in a copper matrix and hot pressed in a spark plasma sintering process with various binding materials at temperatures slightly lower than the melting temperature of copper $[2,3]$. The binding elements form carbides during the sintering process, aiding in the bonding of diamond and copper particles, which otherwise have a lack of affinity [4].

The copper diamond grade tested in the MultiMat experiment, CuCD RHP3434, developed by RHP Technology, consists of a 50-50 volume percentage distribution of copper and diamond, with a measured porosity of approximately $8 \%$, determined by calculating the expected density of the mixture and measuring the actual density of the composite material. Microscopy images of the CuCD RHP3434 tested in this study, with diamond particles dispersed in a copper matrix, are shown in Figure 1.

$\mathrm{CuCD}$ is of particular interest in the field of beam intercepting devices due to the combination of properties provided by the two main material constituents-copper contributes to the thermal and electrical conductivity of the material, while the diamond particles further improve thermal conductivity and aid in reducing the density and the coefficient of thermal expansion [5]. When compared with Inermet180, CuCD has been identified as a more robust material which can withstand a higher beam intensity without severe damage and need for replacement [6]. One of the main limitations of the material is the degradation of the diamondcopper boundary at temperatures above $250-300^{\circ} \mathrm{C}$, a result of the mismatch in the coefficient of thermal expansion between the two materials causing the detaching of diamonds from the copper matrix. This results in an irreversible change in material properties, such as a reduction in thermal diffusivity, which decreases with each thermal cycle above $250^{\circ} \mathrm{C}$ [7].

The material is one of several materials tested in the MultiMat experiment, conducted at CERN's HiRadMat (high radiation to materials) facility in 2017 [8-11], which uses beams from the super proton synchrotron (SPS) accelerator to test components and materials exposed to extreme conditions in CERN's accelerator facility. The MultiMat experiment, or HRMT36, was conducted in October 2017 and aimed to offer a reusable platform for testing materials under high-intensity beam impacts $[6,7]$. The testbench, shown in Figure 2, hosted a number of target stations, each having a length of $1 \mathrm{~m}$, setup on a rotatable barrel equipped with a Geneva mechanism allowing the movement of each target station into the shooting position to be impacted by the incoming particle beam. Tested specimens were equipped with thermal probes and strain gauges and placed on graphitic supports. Other materials tested in the MultiMat experiment included heavy alloys such as titanium zirconium molybdenum, Inermet180 and tantalum tungsten, carbide-graphite composites, silicon

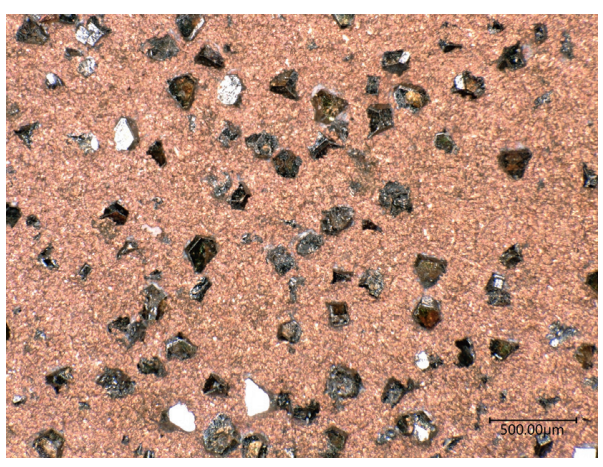

Figure 1: Microscopy image of CuCD RHP3434, with diamond particles dispersed in a copper matrix.

carbide, and carbon foams [13-16]. The pulse intensity ranged from 1 to 288 bunches, with a typical bunch intensity of $1.3 \times 10^{11}$ protons. Different types of beam impact were tested in the experiment, namely, axially centred impacts (resulting in a dynamic longitudinal response), offset impacts (resulting in an additional flexural response), and grazing impacts (for the testing of material coatings).

\section{Material Characterisation}

A material characterisation campaign was carried out at the CERN laboratories to build an elasto-plastic material model for the CuCD RHP3434 grade, comprising of the following:

(i) A thermomechanical characterisation consisting of temperature-dependent measurements for density, specific heat capacity, conductivity, and coefficient of thermal expansion

(ii) An impulse excitation technique test, for the computation of elastic properties (namely, Young's modulus, shear modulus, and Poisson's ratio) at room temperature

(iii) A four-point bending test at room temperature, for the analysis of the stress-strain behaviour of the material, including plasticity

3.1. Thermomechanical Characterisation. A thermomechanical characterisation campaign was conducted, measuring material properties as a function of temperature. Measurements were made for the coefficient of thermal expansion, density, specific heat capacity, and conductivity of the material. The measurements were limited up to a temperature of approximately $300^{\circ} \mathrm{C}$, above which the boundary between the diamond particles and the copper matrix was found to degrade irreversibly, resulting in a regression in thermal properties [7]. Note that the error bars shown in Figures 3-6 refer to the standard deviation between measurements for different samples.

For the measurement of the linear coefficient of thermal expansion, $\alpha$, a Netzsch DIL 402E dilatometer [17] was utilised to measure the expansion (in the heating phase) and shrinkage (in the cooling phase) of CuCD specimens, following the ASTM E228-17 standard "Standard Test 


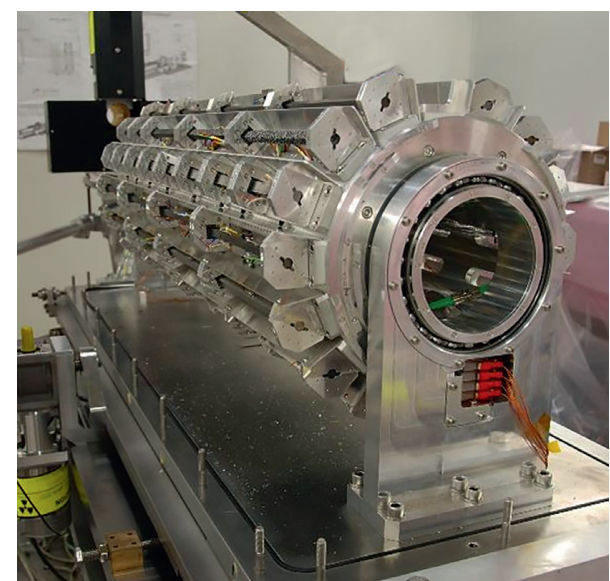

FIgURE 2: Rotatable barrel and mounted target stations in the HRMT36 experiment testbench.

Method for Linear Thermal Expansion of Solid Materials with a Push-Rod Dilatometer" [18]. Measured values for the linear coefficient of thermal expansion are shown in Figure 3.

Initial tests for the calculation of density, $\rho$, were carried out using the Archimedes principle, where the specimen is immersed in a liquid and its volume is determined by the displacement of liquid. The mass of the specimen can then be measured to calculate the density [19]. Due to the high porosity of the $\mathrm{CuCD}$ grade being tested, the alcohol used in the experiment infiltrates the pores, resulting in an overestimation of the density calculated. It was therefore deemed preferable to calculate the density from measurements of the specimens' dimensions and weight. Five specimens of varying dimensions were measured at three different points along their length. The dimensions were averaged, and the density was calculated for each specimen. The results for the five measured specimens were similarly averaged to achieve a final value for density at room temperature $\left(\rho_{0}\right)$, equal to $5700 \mathrm{~kg} \cdot \mathrm{m}^{-3}$. Taking into consideration the volumetric coefficient of thermal expansion $\beta=3 \alpha$, the density can be expressed as a function of temperature by considering temperature-dependent measurements for the coefficient of thermal expansion, as shown in equation (1), where $\rho_{0}$ is the initial density and $T_{0}$ is the initial temperature, i.e., room temperature. The temperature-dependent measurements for the density of the CuCD RHP3434 grade are shown in Figure 4.

$$
\rho\left[\mathrm{kgm}^{-3}\right]=\frac{\rho_{0}}{1+\beta\left(T-T_{0}\right)} .
$$

For the measurement of the specific heat capacity $\left(C_{p}\right)$, a differential scanning calorimeter (DSC) was used. With such a setup, the specific heat capacity was determined via the ratio method [20]. For each test sample, three tests must be performed; the first test being a "baseline" which records system characteristics and allows removal of the system bias from experimental data. After a repeatable baseline has been recorded, a reference material must be tested, following which the experimental sample is tested in exactly the same

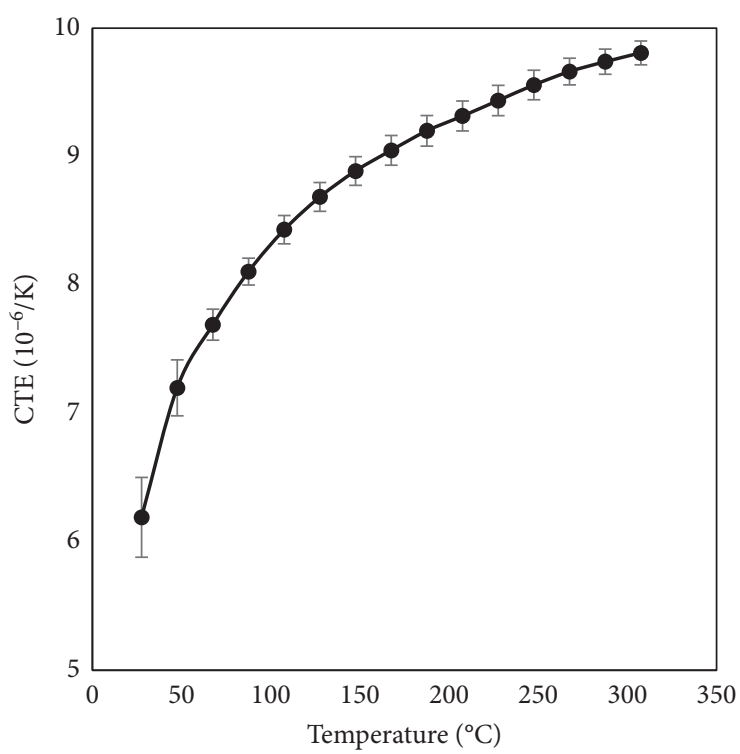

Figure 3: Linear coefficient of thermal expansion of $\mathrm{CuCD}$ RHP3434.

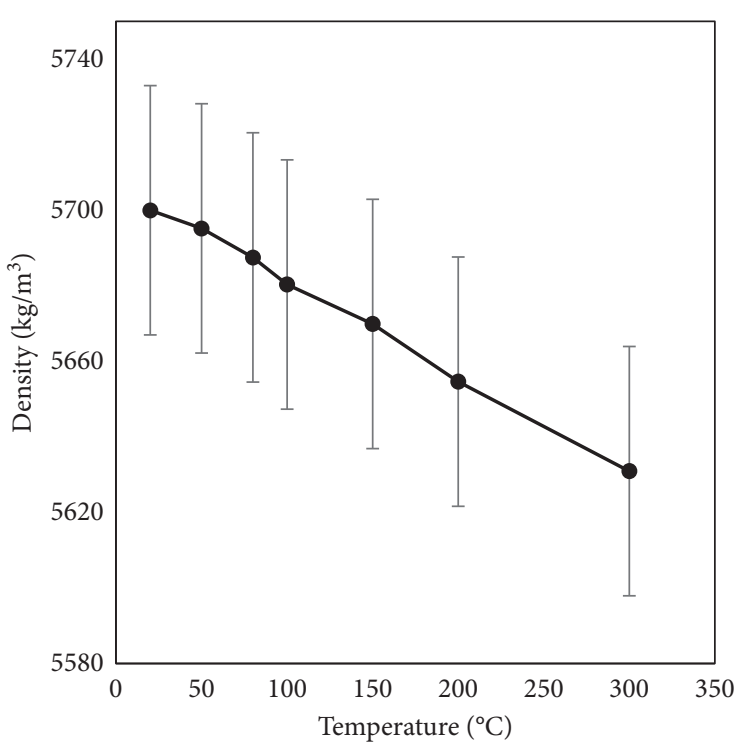

Figure 4: Density of CuCD RHP3434.

way as reference $[21,22]$. The specific heat capacity measurements for the CuCD RHP3434 grade are shown in Figure 5.

The thermal diffusivity was measured by using a laser flash apparatus (LFA), which shoots a short laser pulse onto one of the specimen's faces and measures the temperature on the opposite face with the use of an optical pyrometer. Knowing the specimen's thickness, the thermal diffusivity across a range of temperatures can be computed from these readings. The conductivity, $k$, was then found by multiplying the diffusivity (in $\mathrm{m}^{2} \cdot \mathrm{s}^{-1}$ ), the density, and the specific heat capacity [23]. The calculated thermal conductivity results are shown in Figure 6. 


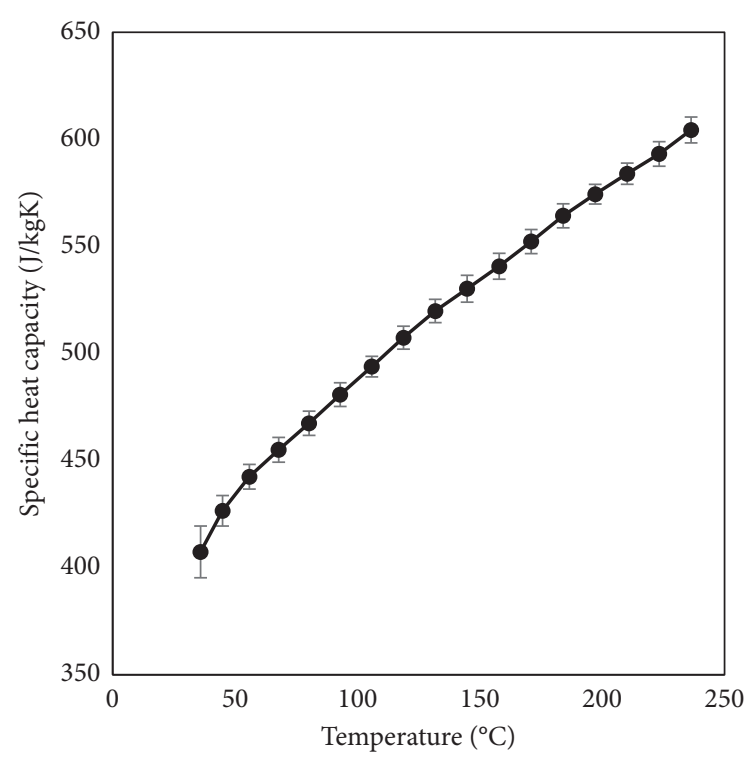

Figure 5: Specific heat capacity of CuCD RHP3434.

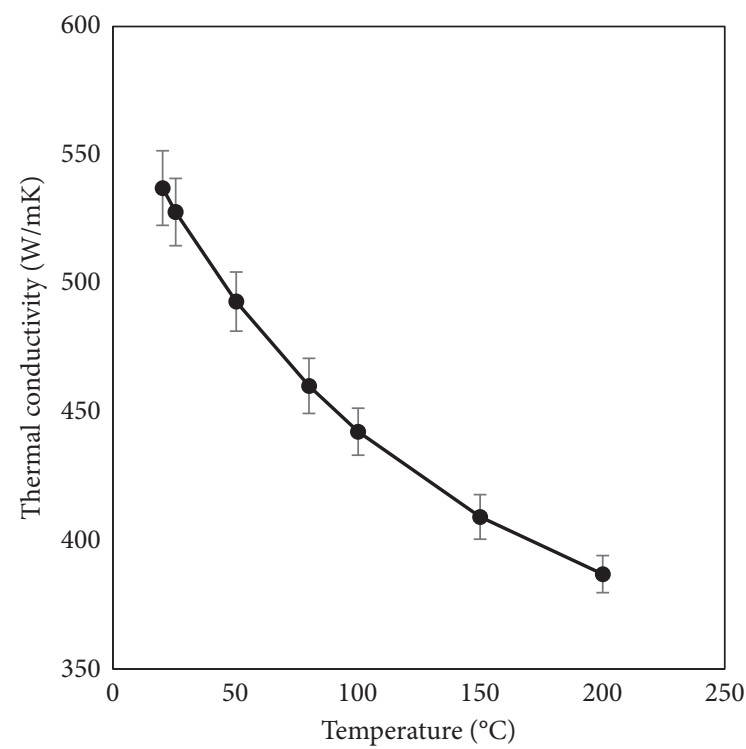

Figure 6: Thermal conductivity of CuCD RHP3434.

Measured values for the CTE, density, specific heat capacity, and thermal conductivity are summarised at room temperature in Table 1.

3.2. Impulse Excitation Technique Test. An impulse excitation technique test was conducted to determine the elastic properties of the CuCD RHP3434 grade. As will be discussed in this section, the expected flexural and torsional frequencies to be measured in the test were first calculated in accordance with industry standards, following which the test was performed and the excited modes of interest identified. This was followed by a preliminary modal finite element analysis conducted with estimated elastic constants from measurements on other $\mathrm{CuCD}$ grades. The elastic properties
TABLE 1: CuCD RHP3434 material properties at room temperature.

\begin{tabular}{lcccc}
\hline Property & Symbol & Value & $\begin{array}{c}\text { Standard } \\
\text { uncertainty }\end{array}$ & Units \\
\hline Density & $\rho$ & 5700 & \pm 33 & $\mathrm{~kg} \cdot \mathrm{m}^{-3}$ \\
Specific heat capacity & $C_{p}$ & 407 & \pm 12 & $\mathrm{~J} \cdot \mathrm{kg}^{-1} \mathrm{~K}^{-1}$ \\
$\begin{array}{l}\text { Thermal conductivity } \\
\text { Linear coefficient of }\end{array}$ & $k$ & 537 & \pm 15 & $\mathrm{~W} \cdot \mathrm{m}^{-1} \mathrm{~K}^{-1}$ \\
thermal expansion & $\alpha$ & 6.19 & \pm 0.2 & $10^{-6} \mathrm{~K}^{-1}$ \\
\hline
\end{tabular}

of the material were then optimised to the frequencies measured in the IET test.

The impulse excitation technique test is a dynamic, nondestructive material characterisation technique which measures the resonant frequencies of a material, which are subsequently used to calculate the material's elastic constants at room temperature or at elevated temperatures [24]. The measurement consists of hitting the sample with a hammer and recording the induced vibration modes by means of a microphone. The vibrational signal measured in the time domain is then converted to the frequency domain by a fast Fourier transform (FFT), following which the resonant frequencies can be determined, allowing for the calculation of the elastic properties of the material, taking into consideration the dimensions at hand.

Different frequency modes (resonant frequencies) can be excited in the specimen and are mainly dependent on the position of the support wires, the mechanical impulse, and the measuring device. With regard to the elastic properties of the material, the two most important resonant frequencies are the flexural and torsional frequencies, which are mostly dependent on Young's and shear moduli for isotropic materials. The specimen's elastic properties can be calculated by considering the dimensions and geometry, weight, and the measured resonant frequencies in accordance with the ASTM E1876-15 "Standard Test Method for Dynamic Young's Modulus, Shear Modulus, and Poisson's Ratio by Impulse Excitation of Vibration" [25, 26].

The natural flexural frequency, denoted as $f_{f}$ is characteristic of Young's modulus, E. For the measurement of this frequency, the specimen is supported at the nodes (where the amplitude of vibration is zero) and is excited at one of the antinodes (i.e., one of the ends of the rod) in order to provoke maximum vibration, as shown in Figure 7(a). Following the flexural frequency measurement, Young's modulus can be calculated by

$$
E=0.9465\left(\frac{m \cdot f_{f}^{2}}{b}\right)\left(\frac{L^{3}}{h^{3}}\right) R_{f},
$$

where $m$ is the mass, $b, h$, and $L$ are the width, thickness, and length of the specimen, $f_{f}$ is the measured flexural frequency, and $R_{f}$ is a geometrical correction factor which represents the aspect ratio of the specimen, depending on the thickness and length of the specimen.

As can be seen in equation (2), the mass and dimensions of the sample need to be measured accurately for reliable calculation of Young's modulus. Similarly, the natural torsional frequency, denoted as $f_{t}$, is characteristic of the 


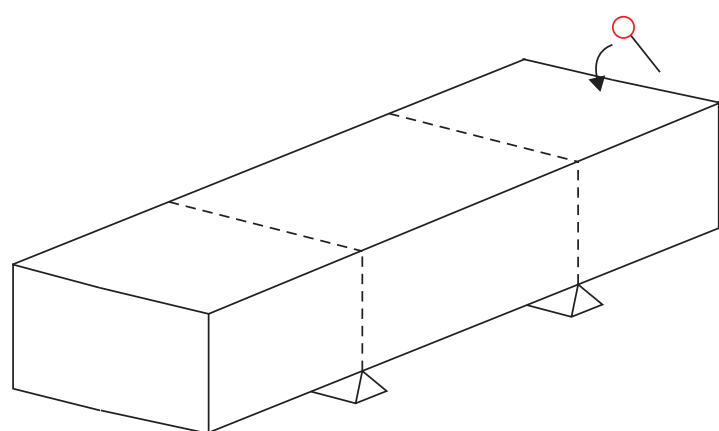

(a)

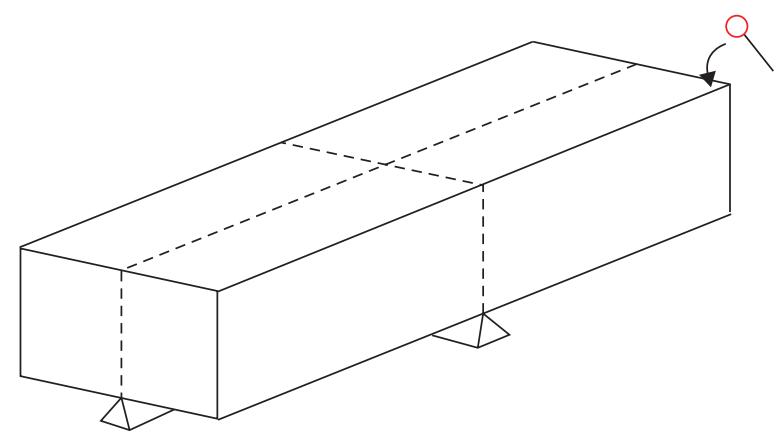

(b)

Figure 7: IET setup for flexural (a) and torsional (b) mode excitation.

shear modulus. In this case, the specimen is supported along both longitudinal and transverse axes, as can be seen in Figure 7(b), while the mechanical excitation is performed at one corner, inducing a twisting motion in the beam, rather than flexing it. Following the torsional frequency measurement, the shear modulus can be calculated by the following:

$$
G=\frac{4 \cdot L \cdot m \cdot f_{t}^{2}}{b \cdot h} R_{t}
$$

where $f_{t}$ is the measured torsional frequency and $R_{t}$ is a geometrical correction factor. Poisson's ratio, $v$, is calculated by $v=[(E / 2 G)-1]$, and the material damping can also be characterised by considering the logarithmic decrement of the decaying amplitude of vibration.

While the solutions defined by equations (2) and (3) provide adequate results for elastic properties, a numerical approach encompasses the triaxiality of the problem and can allow for the optimisation of the elastic constants, allowing for the reverse engineering of these values from the measured frequencies with great accuracy. In addition, a numerical approach has the benefit of allowing the calculation of elastic constants for anisotropic bodies. The following section describes the numerical approach adopted in this study to determine the elastic properties of the tested $\mathrm{CuCD}$ grade. For a specimen with a square cross section and made of an isotropic material, measurements of resonant frequencies in different transverse planes (referred to as inplane and through-plane) are identical since the orientation of the specimen does not have an effect on the material properties. Whilst the method described in the following section refers to isotropic bodies-as is the case for $\mathrm{CuCD}$ - it can easily be extended to anisotropic bodies by considering flexural and torsional mode measurements for both in-plane and through-plane conditions.

The finite element analysis optimisation was implemented in the software ANSYS Workbench [27] using a modal FEM analysis which, for a defined geometry, calculates the resonant frequencies for the given elastic properties and density. Arbitrary elastic properties are initially input, which are then optimised with the resonant frequencies measured from the experiment. During preprocessing, the geometry is divided into sub-blocks, allowing the definition of several lines and surfaces which can be constrained to force the excitation of a particular resonance mode. The density value input in the simulation is also of particular importance, given that the frequency response is directly dependent on this property (for a given set of properties and dimensions, a higher density results in lower frequencies and vice versa). The experimentally derived density values detailed in the previous sections of this study were used for the $\mathrm{CuCD}$ grade in question (i.e., $5700 \mathrm{~kg} / \mathrm{m}^{3}$ at room temperature).

If the specimen tested has a rectangular cross section, two possible flexural planes are present. During the simulation setup, the flexural mode in the direction of interest (i.e., the direction excited in the experiment) is forced by defining a symmetrical plane in this direction. This ensures movement in the direction perpendicular to the symmetrical plane is restricted. For the excitation of the torsional mode, the longitudinal and transverse axes are fixed (i.e., displacement equal to zero), blocking movement of these lines in all directions. A constraint equation is applied to the two faces at the extremities of the specimen, promoting a natural torsional behaviour where the two faces rotate in opposite directions. The relevant boundary conditions for the two analyses are shown in Figure 8.

Once the initial simulations are setup and completed (utilising approximate values for the elastic constants), the resulting first flexural and torsional frequencies are parametrised and optimised to the experimental measurements. The variables influencing the two frequencies (flexural and torsional) are Young's modulus and Poisson's ratio (the shear modulus is calculated from the other properties). A range of acceptable results is defined for each parameter.

The IET test was carried out on a specimen with dimensions $\quad 247 \times 12.41 \times 10.35 \mathrm{~mm}^{3} \quad$ (length $\times$ width $\times$ thickness). A spectrogram showing the intensity of the excited frequencies with respect to time in the tested specimen, setup in the configuration shown in Figure 7(a), is shown in Figure 9. As shown in the spectrogram, there are various excited frequencies in the measured range. In order to identify the modes of interest, using Young's modulus of $208 \mathrm{GPa}$ and Poisson's ratio of 0.22 as a first approximation, the first flexural and torsional modes were calculated by equations (2) and (3), as shown in Table 2. This allows for the identification of the first torsional and flexural frequencies from the various unwanted frequencies excited in the experiment. 


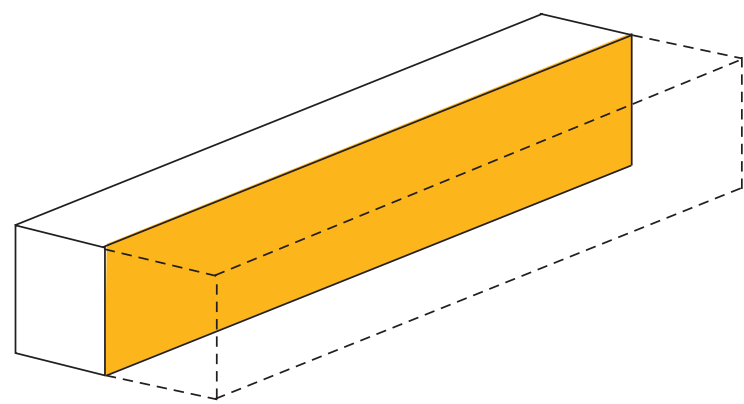

(a)

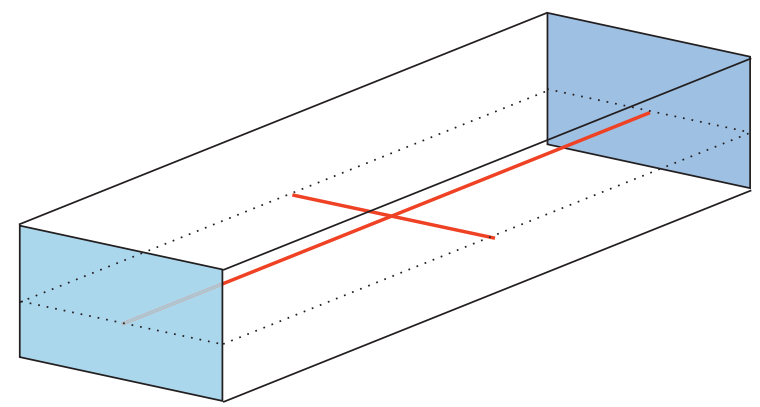

(b)

FIGURE 8: Boundary conditions forcing mode of interest: (a) symmetry plane promoting flexural vibration and (b) fixed axes promoting torsional vibration and a constraint equation limiting motion of the two extremities of the specimen to rotate in opposite directions.

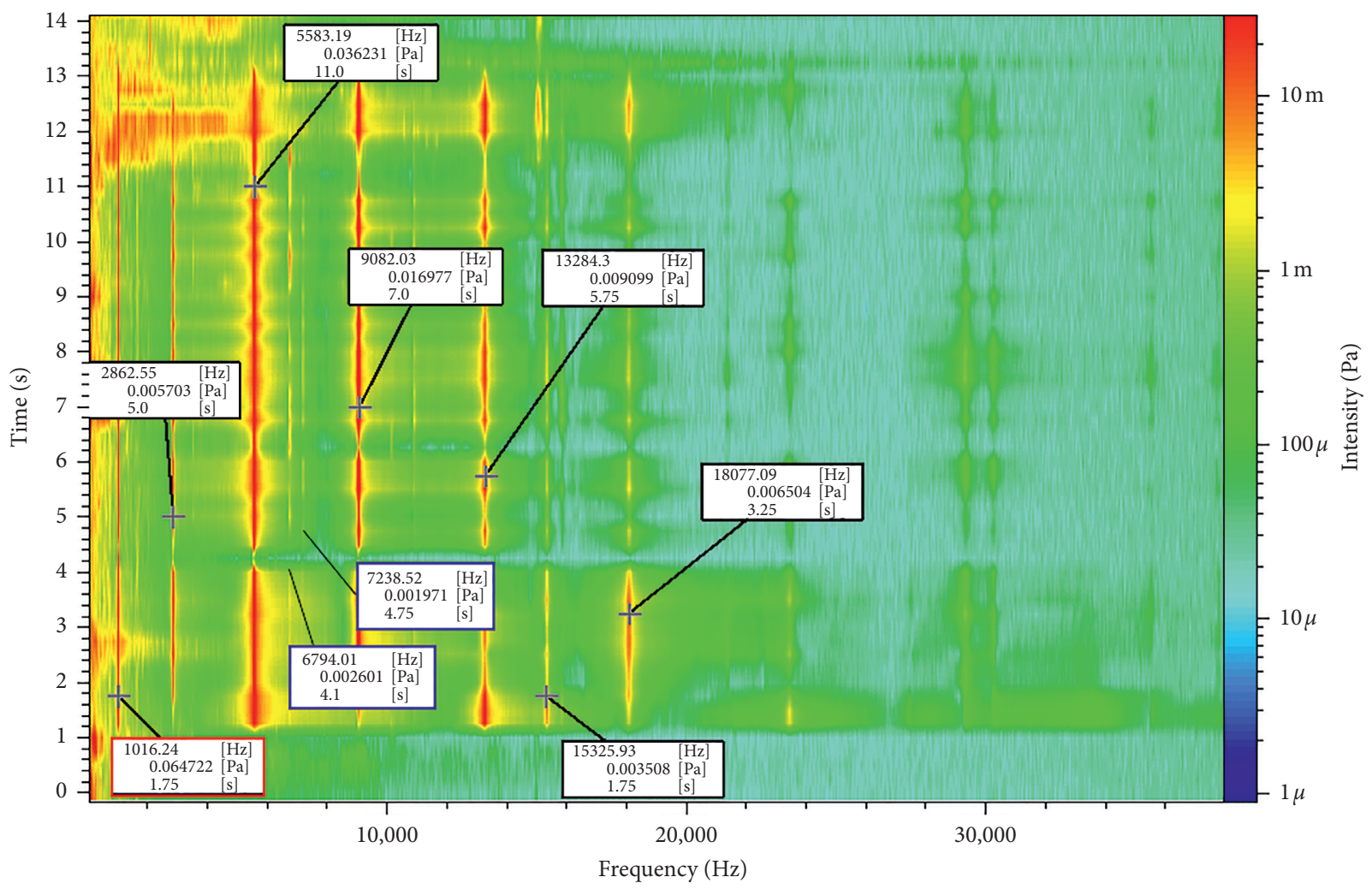

FIgURE 9: Spectrogram showing the IET test flexural frequency measurement as a function of time.

TABLE 2: Calculated flexural and torsional modes for positions A and B with initial estimates of elastic properties $(E=208 \mathrm{GPa}$ and $\nu=0.22$ ).

\begin{tabular}{lcc}
\hline $\begin{array}{l}\text { Specimen dimensions, } \\
\text { length } \times \text { width } \times \text { thickness }\end{array}$ & $\begin{array}{c}\text { Flexural } \\
\text { mode }(\mathrm{kHz})\end{array}$ & $\begin{array}{c}\text { Torsional } \\
\text { mode }(\mathrm{kHz})\end{array}$ \\
\hline $247 \times 12.41 \times 10.35 \mathrm{~mm}^{3}$ & 1.047 & 7.054 \\
\hline
\end{tabular}

From the experimental results, the first flexural frequency can be easily identified, with a peak at $1.016 \mathrm{kHz}$. The slender nature of the specimen made it difficult to excite the torsional vibration; however, two possible frequencies corresponding to the torsional mode were identified in the flexural measurement at $6.794 \mathrm{kHz}$ and $7.238 \mathrm{kHz}$ (two faint lines between the $5.583 \mathrm{kHz}$ and $9082 \mathrm{kHz}$ readings).

Following the identification of the first flexural mode and the two possible torsional modes, the initial estimates for Young's modulus and Poisson's ratio-208 GPa and 0.22, respectively-were used as a primary input, along with the density of the material, in the optimisation procedure. A first estimation of the flexural and torsional modes was computed for the given material properties. The first flexural and torsional modes determined by the modal analyses are shown in Figure 10. As can be seen, additional 'undesired' modes, such as the first longitudinal harmonic, can also be excited since movement in this direction is unrestricted. 


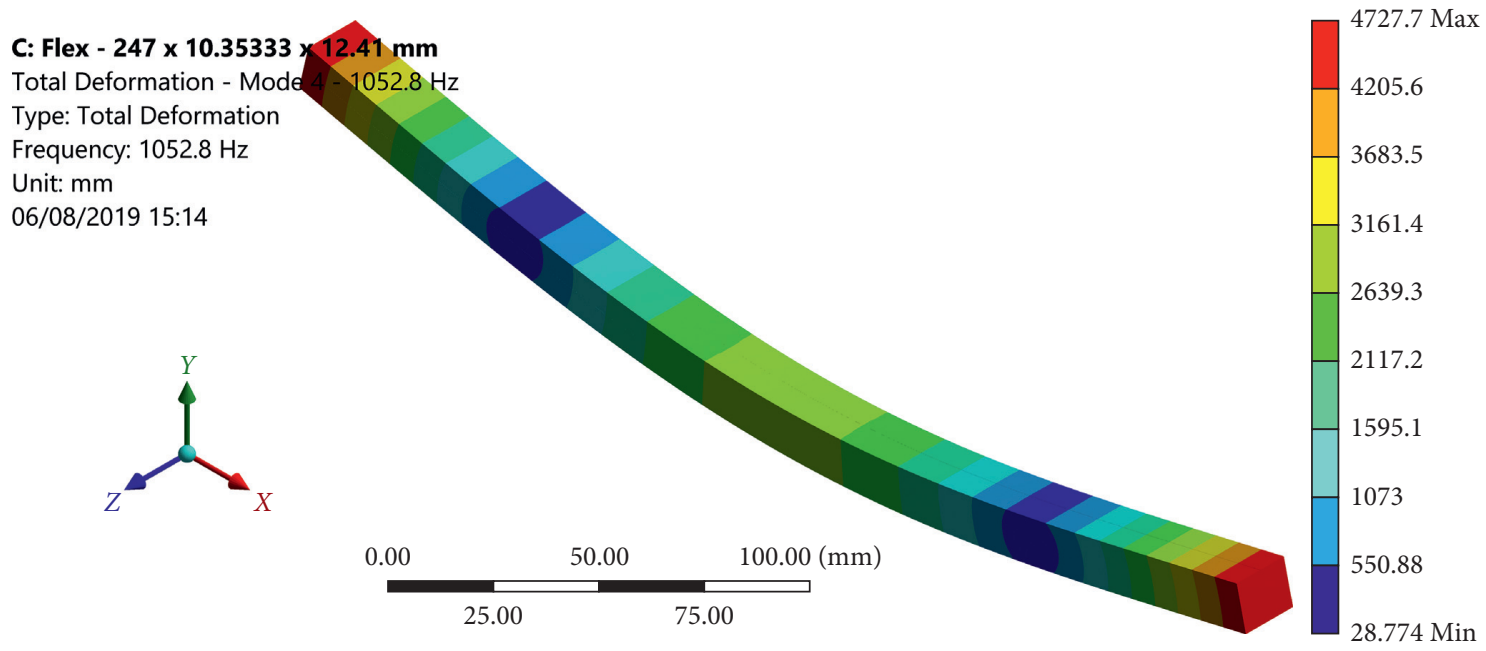

(a)

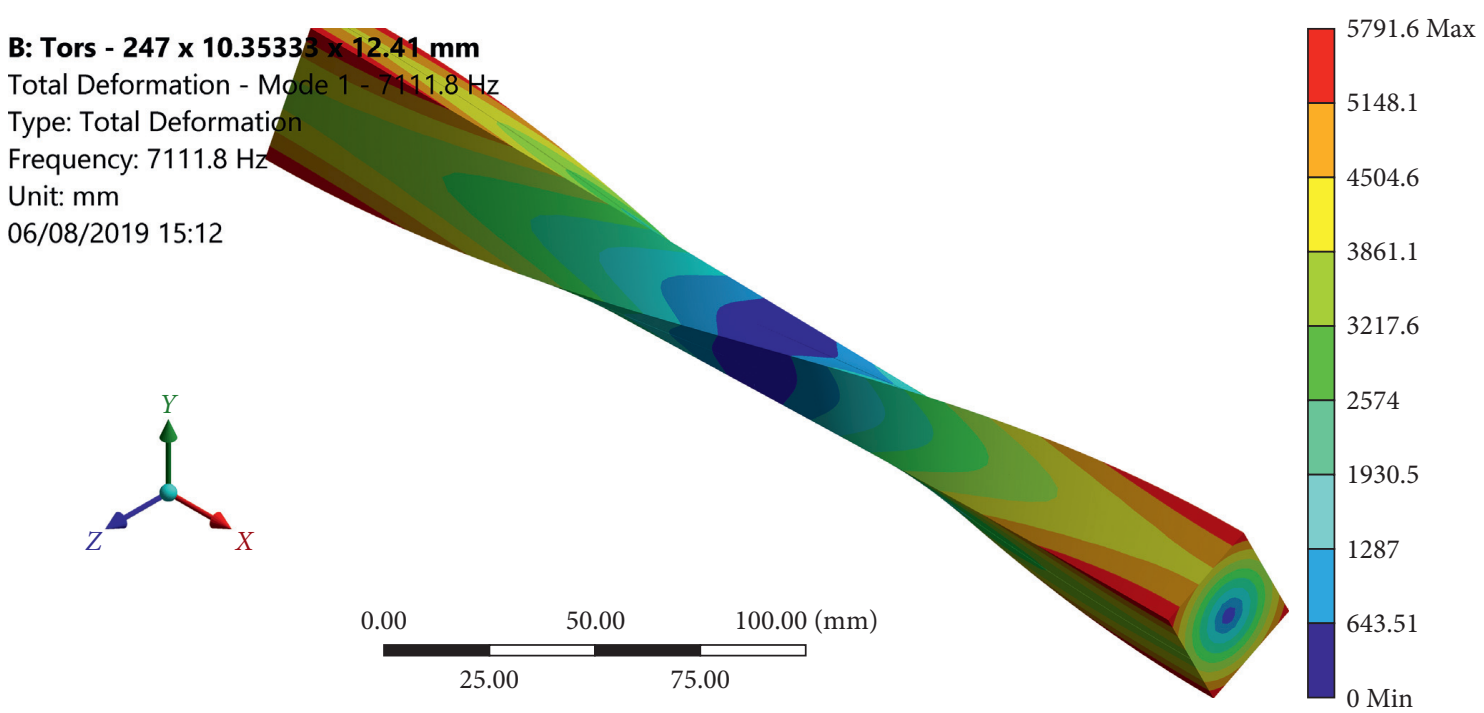

(b)

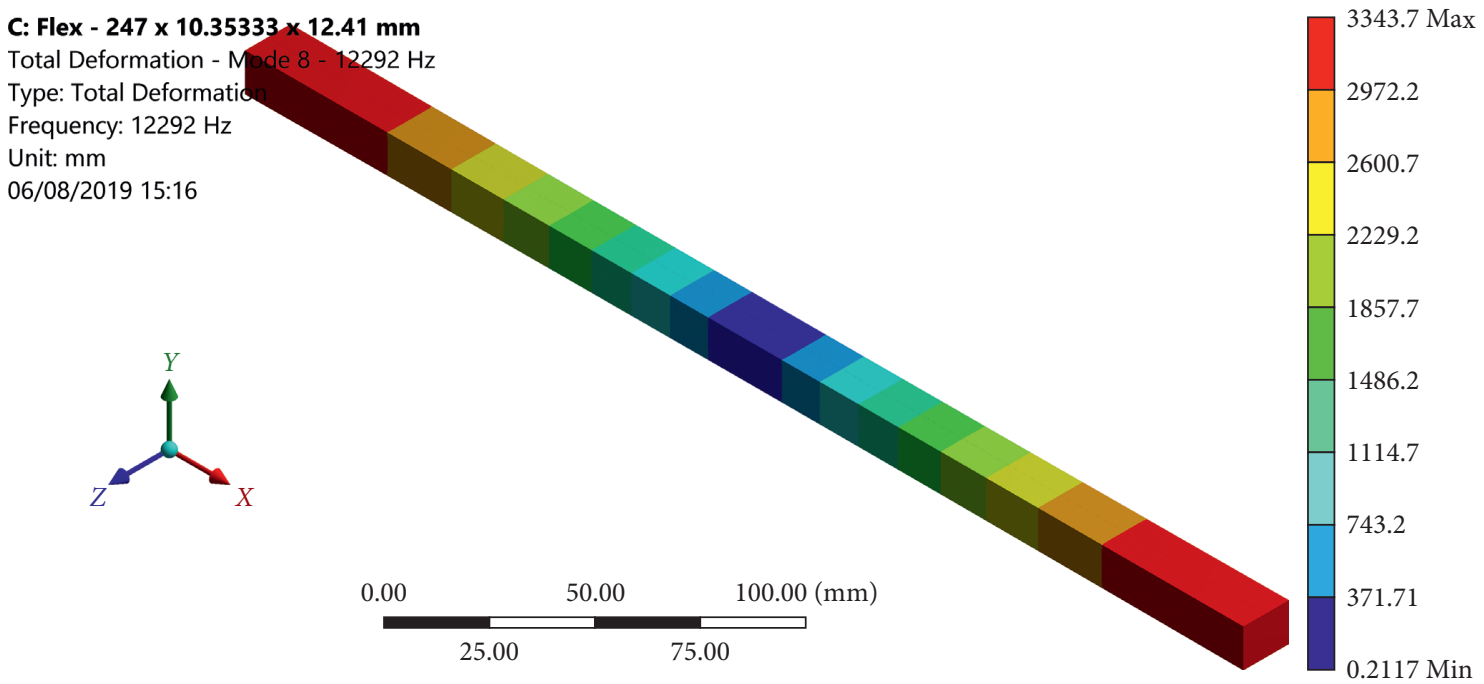

(c)

Figure 10: First flexural and torsional modes simulated with estimated material properties $(\mathrm{a}, \mathrm{b})$ and additional modes such as the first longitudinal one (c). 
Following the initial modal analysis with estimated properties, the computed frequencies were parametrised and optimised with the frequencies measured in the experiment. Young's modulus was constricted to a range between $187 \mathrm{GPa}$ and $229 \mathrm{GPa}$, while Poisson's ratio was restricted to values ranging from 0.1 to 0.3 . The optimisation procedure varies these two values, attempting to match the computed frequencies with the measured values in the experiment.

The relatively minor difference $(11 \%)$ in the two measured frequencies considered for the torsional mode resulted in a significant effect on the computed Poisson's ratio. For $f_{f}=1.016 \mathrm{kHz}$ and $f_{t}=6.794 \mathrm{kHz}$, Young's modulus is computed at $193.7 \mathrm{GPa}$, with Poisson's ratio of 0.248 , while for $f_{f}=1.016 \mathrm{kHz}$ and $f_{t}=7.238 \mathrm{kHz}$, Young's modulus is calculated at $193.6 \mathrm{GPa}$, with Poisson's ratio of 0.104 . Considering previous data on similar $\mathrm{CuCD}$ grades, the value of $\nu=0.248$ was deemed to be the physically valid one and retained for the material model, along with a rounded Young's modulus value of $194 \mathrm{GPa}$ [13]. The final values for Young's modulus and Poisson's ratio are shown in Table 3.

3.3. Plasticity-Four-Point Bending Experiment. To model the material behaviour beyond the elastic regime, a four-point bending test was performed, allowing for the implementation of a multilinear hardening model in the numerical analyses. A Zwick/Roell Z400 universal testing machine was used for the four-point flexural test in accordance with the ASTM C651 standard "Flexural Strength of Carbon and Graphite by Four-Point Loading" [28]. With this experimental setup, as shown in the schematic in Figure 11, the bending force is applied at two points along the length of the specimen, which is supported at its extremities. Specimens with an average length of $30 \mathrm{~mm}$, width of $5.3 \mathrm{~mm}$, and thickness of $5.1 \mathrm{~mm}$ were tested with such a setup.

In the experiment, the bending force is progressively increased, with the corresponding induced axial strain in the specimen measured with a strain gauge glued to its surface. The maximum bending moment $M$ is deduced from the force $F$ and the distance between the load application and the supports $L_{1}$, by $M=\left(L_{1} F / 2\right)$. In the linear elastic domain, the stress distribution through the sample thickness can be assumed to be linear, and the maximum can be calculated by $\sigma=(M h / 2 I)$, where $I$ is the flexural moment of inertia and $h$ is the sample thickness. However, the force-strain relation measured in the experiment is nonlinear, indicating that there is a nonlinear stress distribution across the thickness of the specimen, as shown in Figure 12. The maximum stress thus has to be calculated taking into consideration this nonlinearity. For small curvatures, the axial strain can be considered to be linearly distributed through the thickness [7].

The stress must therefore be calculated out of the general moment-strain relationship. The moment can be expressed as an integration of the stress distribution through the thickness by

$$
M=b \int_{-h / 2}^{h / 2} \sigma_{z}(x) x \mathrm{~d} x
$$

TABle 3: Young's modulus and Poisson's ratio results determined by the IET optimisation procedure.

\begin{tabular}{lcccc}
\hline Property & Symbol & Value & Uncertainty & Units \\
\hline Young's modulus & $E$ & 194 & \pm 32 & $\mathrm{GPa}$ \\
Poisson's ratio & $v$ & 0.248 & \pm 0.002 & - \\
\hline
\end{tabular}

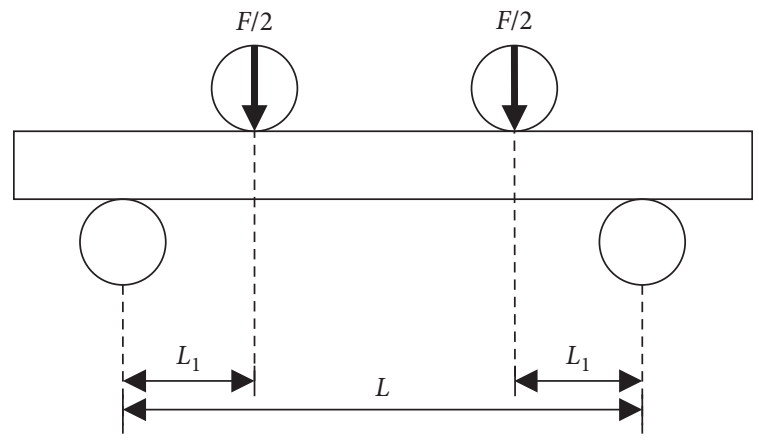

FIgURE 11: Four-point bending experimental setup.

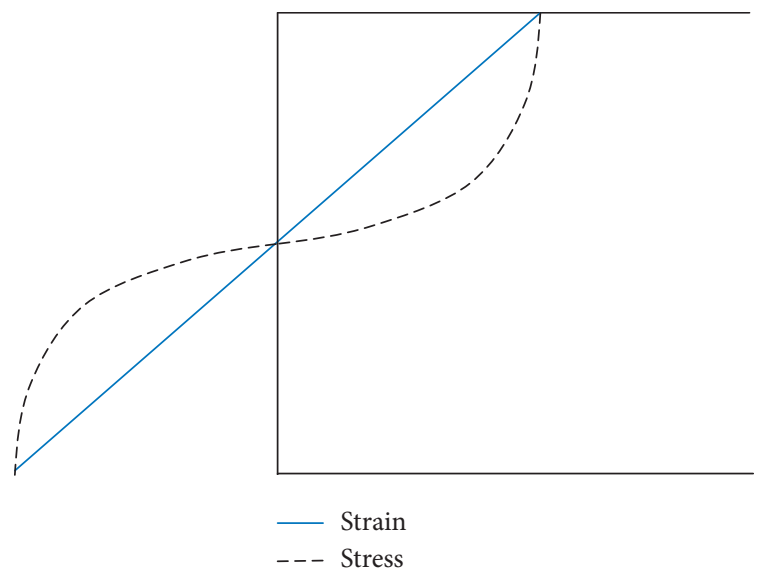

Figure 12: Axial strain (linear) and stress (nonlinear) distribution through the sample thickness.

where $x$ is the vertical coordinate from the neutral axis, $b$ is the specimen's width, and $h$ is its thickness (height, i.e., along $x$ ). The moment can be expressed in terms of the measured strain as follows:

$$
M\left(\varepsilon_{\max }^{\mathrm{tot}}\right)=2 b \int_{0}^{h / 2} \sigma_{z}\left(\frac{2 x}{h} \varepsilon_{\max }^{\mathrm{tot}}\right) x \mathrm{~d} x,
$$

where $\sigma_{z}\left((2 x / h) \varepsilon_{\max }^{\text {tot }}\right)$ indicates the stress-strain curve which can be numerically derived, given the applied moment $M\left(\varepsilon_{\max }^{\text {tot }}\right)$ and the measured strain $\varepsilon_{\max }^{\text {tot }}$. The problem can be solved for each measured value of force and strain, resulting in a piecewise stress-strain relationship, i.e., a multilinear hardening curve describing the plastic behaviour of the material, shown in Figure 13. The material can be seen to quickly lose linearity and undergoes a significant amount of plastic deformation as a result of the highly ductile copper matrix. 


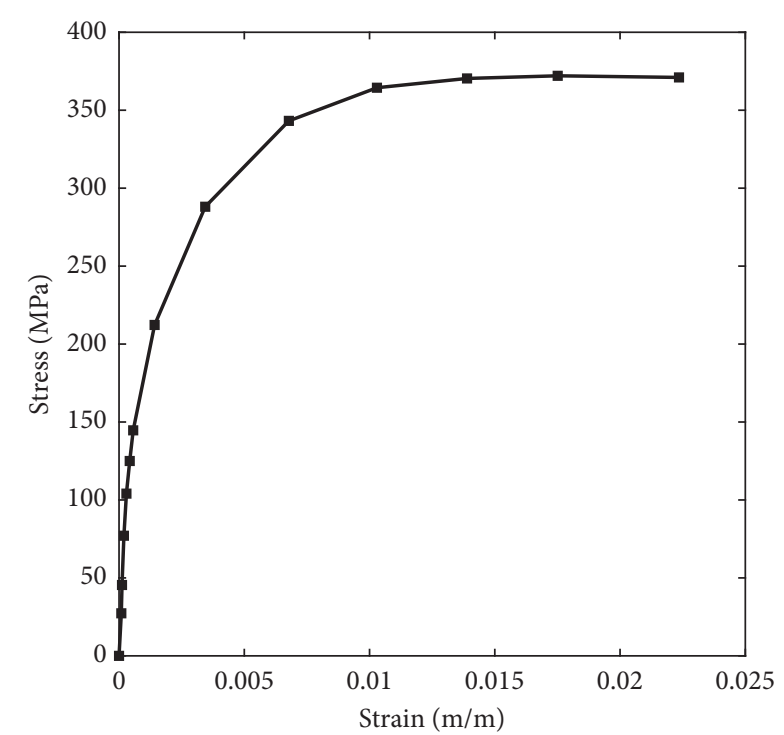

FIgURE 13: Stress-strain diagram for CuCD RHP3434 obtained from the four-point bending test.

One aspect which is not considered in the model is the compression behaviour of the material, which is assumed to be identical to that in tension. This assumption may not be valid at high compressive strains, at which point the diamond particles may come in contact. In contrast, when the material is loaded in tension, the bond between the diamond particles and the copper matrix may be lost.

\section{Benchmarking of the Material Model}

4.1. HRMT36: The MultiMat Experiment. CERN's HiRadMat facility is used to test materials used for components exposed to extreme conditions in CERN's accelerator complex [10]. Upcoming LHC upgrades such as the highluminosity LHC (HL-LHC) upgrade and the proposed Future Circular Collider (FCC) require an increase in the beam energy and thus bring the need for high-performing materials. This is especially true for use in components at risk of exposure to accidental beam impacts, such as collimators and other beam intercepting devices [29].

Experiments conducted in the HiRadMat facility include tests on full-scale collimator jaws [29-31] as well as specimens with simple geometries, allowing the benchmarking of numerical results obtained through numerical analyses $[9,14]$. The HRMT36 "MultiMat" experiment, conducted in October 2017, was designed to offer a reusable platform to test a wide range of novel materials developed for beam intercepting devices, impacted by particle beams with energy densities in the range of those experienced in the HL-LHC $[32,33]$.

The test bench hosted 16 target stations, mounted on a rotatable barrel, with each station having a total length of $1 \mathrm{~m}$. The material specimens were mounted in series and had lengths of 120 or $247 \mathrm{~mm}$, with cross sections varying from $8 \times 8$ to $12 \times 11.5 \mathrm{~mm}^{2}$. 18 different materials were tested in total. The acquisition system included electrical strain gauges and temperature probes placed on the material specimens, along with remote instrumentation including a laser
Doppler vibrometer (LDV) oriented towards the top face of the specimens and a side-mounted radiation-hard highdefinition camera [12]. The dimensions for $\mathrm{CuCD}$ specimens tested and typical positions of strain gauges and thermal probes are shown in Figure 14. The target station hosting the CuCD RHP3434 samples consisted of 4 specimens in series, each having a length of $247 \mathrm{~mm}$ (with a $3 \mathrm{~mm}$ space inbetween specimens) and a square cross section with an edge length of $10 \mathrm{~mm}$. All four specimens were equipped with longitudinal strain gauges to measure dynamic phenomena induced by the particle beam impacts.

Other than copper diamond (of which two grades from two different suppliers were tested), various materials and material grades were tested in the MultiMat experiment, including 4 grades of MoGr, 3 different coatings ( $\mathrm{Cu}, \mathrm{Mo}$, and $\mathrm{TiN}$ ), and novel carbon-based materials such as highly ordered pyrolytic graphite (HOPG) and titanium-graphite (TG-1100). The pulse intensity to which specimens were subjected to ranged from 1 to 288 bunches, with a typical bunch intensity of $1.3 \times 10^{11}$ protons, along with nominal beam RMS sizes of $0.25 \times 0.25$, $0.5 \times 0.5$, and $2 \times 2 \mathrm{~mm}^{2}$. The three different types of impact tested were axially centred impacts, offset impacts, and grazing impacts (where a coating was present).

4.2. Modelling and Benchmarking with Experimental Results. The material model for the CuCD RHP3434 grade, consisting of the measured thermomechanical temperaturedependent properties (Figures 2-5, summarised in Table 1), dynamic elastic constants from the IET test (Table 3), and multilinear hardening curve from the four-point bending test (Figure 13), as shown in the previous sections, was included in a simply-coupled, implicit dynamic thermomechanical simulation performed in the FEA software ANSYS. The material is assumed to be isotropic in nature, based on previous study of the material [6], and is assumed to behave in an elasto-plastic manner based on the fourpoint bending test presented in this study. Similar analyses have been performed on other materials tested in the MultiMat experiment, and information on the thermomechanical modelling aspects in such scenarios is detailed in previous studies $[13,15]$. Similarly, analytical solutions for the dynamic response of slender beams subjected to thermal shock have also been studied extensively [34-39].

Figure 15 details the flow of data of the simply-coupled thermomechanical analysis, starting with the FLUKA energy deposition maps which volumetrically map the transfer of energy in the tested material specimens as a result of the particle beam impact [40]. This is used as an input in transient thermal analyses, which compute the temperature in the specimens as a result of the beam impact. The thermal analyses were modelled adiabatically given that the thermal evolution within the specimen is in the order of seconds, compared with the dynamic phenomena observed which are in the order of microseconds [35]. The mesh size used in thermal analyses considered the energy density map bin size-in this case, the mesh consisted of 50 divisions along the longitudinal coordinate and 60 divisions across the cross section, resulting in a model with 379,181 elements. 3D 20-node, high-order thermal 


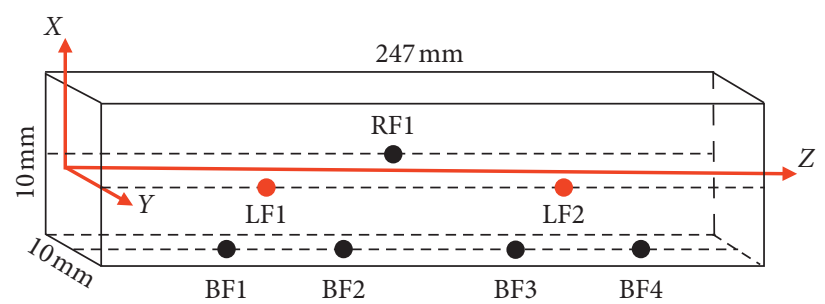

Figure 14: Typical dimensions and position of longitudinal strain gauges (black) and temperature sensors (red) on the specimens. Longitudinal strain gauges are referenced by a BF (bottom face) or RF (right face) notation, and thermal probes are referenced by an LF (left face) notation.

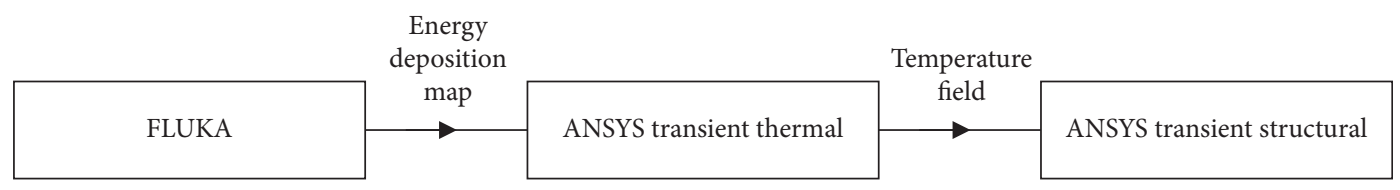

Figure 15: Flowchart detailing flow of data for the weakly coupled thermomechanical analysis.

elements were adopted for this analysis. This provided a good balance between accuracy in thermal gradient computed and computation time. The thermal analysis consisted of two steps: one modelling the energy deposition and the other spanning over a longer time period in order to compute the thermal evolution across the material.

The temperature field generated from the thermal analysis was imported as a thermal load in the transient structural analysis, which models the dynamic phenomena measured in the MultiMat experimental campaign. The structural analysis consists of three steps-the first covering the energy deposition period and consisting of 10 substeps, the second considering the dynamic longitudinal response and covering five longitudinal wave oscillations, and the third considering the flexural response, covering three bending oscillations. The substep for each phase was set accordingly depending on the phenomenon of interest, and the Courant-Friedrichs-Lewy (CFL) condition was considered to determine the appropriate element size, considering the speed of sound of the material and the respective time step. This resulted in a $247 \times 10 \times 10 \mathrm{~mm}^{3}$ model with 15,350 elements and 70,753 nodes. Higher order 3D 20-node solid elements with quadratic displacement behaviour were used for the structural analysis. With regards to boundary conditions, a simply supported configuration was modelled, restricting vertical movement of the bottom edges at the extremities of the specimen, as shown in Figure 16 [13].

The thermomechanical material model adopted in the analysis was built from measurements discussed in the Material Characterisation section. Temperature-dependent values for the linear coefficient of thermal expansion, density, specific heat capacity, and thermal conductivity were adopted as shown in Figures 3-6 in the Thermomechanical Characterisation section. Young's modulus and Poisson's ratio were obtained as described in the Impulse Excitation Technique Test section, while the multilinear hardening curve was found as shown in the Plasticity-FourPoint Bending Experiment section. The material properties at room temperature are summarised in Table 4.

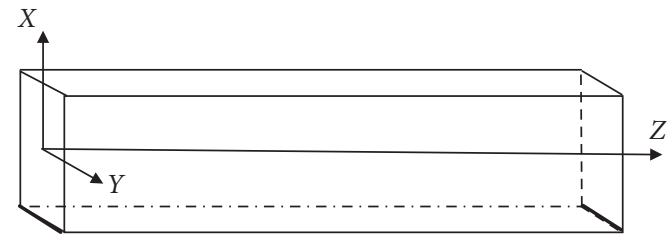

FIGURE 16: Structural model "simply-supported" boundary condition setup (bold edges constrained in X-direction).

TABLE 4: Summary of the material model for CuCD RHP3434 at room temperature.

\begin{tabular}{lccc}
\hline Property & Value & Uncertainty & Units \\
\hline Density & 5700 & \pm 33 & $\mathrm{~kg} \cdot \mathrm{m}^{-3}$ \\
Specific heat capacity & 407 & \pm 12 & $\mathrm{~J} \cdot \mathrm{kg}^{-1} \mathrm{~K}^{-1}$ \\
Thermal conductivity & 537 & \pm 15 & $\mathrm{~W} \cdot \mathrm{m}^{-1} \mathrm{~K}^{-1}$ \\
Coefficient of thermal & 6.19 & \pm 0.2 & $10^{-6} \mathrm{~K}^{-1}$ \\
expansion & 194 & \pm 32 & $\mathrm{GPa}$ \\
Young's modulus & 0.248 & 0.002 & - \\
Poisson's ratio & & & \\
\hline
\end{tabular}

Beam parameters for the two pulses modelled in numerical simulations, such as pulse duration, intensity, transverse beam size $\sigma$, and vertical offset $\eta_{y}$, are shown in Table 5. The first shot had a total intensity of $1.433 \times 10^{11}$ protons (1 bunch) and a vertical offset of $3 \mathrm{~mm}$, while the second shot consisted of a longer, central, 12-bunch shot with a total intensity of $6.880 \times 10^{12}$ protons. Modelling two distinctly different pulses allows one to test the ability of the formulated material model to simulate different scenarios. The calculated energy deposited along the length of the specimens, along with the temperature along the length of the specimens following beam impact (i.e., at $25 \mathrm{~ns}$ for shot 128 and at $300 \mathrm{~ns}$ for shot 132), can be seen in Figure 17. Note that the temperature line was probed along the beam impact position, i.e., at a vertical offset of $3 \mathrm{~mm}$ for shot 128 and along the longitudinal axis of the specimen for shot 132 . 
TABLE 5: Beam parameters for the studied impacts (pulse 128 and pulse 132).

\begin{tabular}{lcccccc}
\hline Pulse no. & Pulse duration (ns) & No. of bunches & Bunch intensity (protons/bunch) & Total intensity (protons) & $\sigma(\mathrm{mm})$ & $\eta_{y}(\mathrm{~mm})$ \\
\hline 128 & 25 & 1 & $1.433 \times 10^{11}$ & $1.433 \times 10^{11}$ & 0.5 & 3 \\
132 & 300 & 12 & $5.733 \times 10^{11}$ & $6.880 \times 10^{12}$ & 0.5 & 0 \\
\hline
\end{tabular}

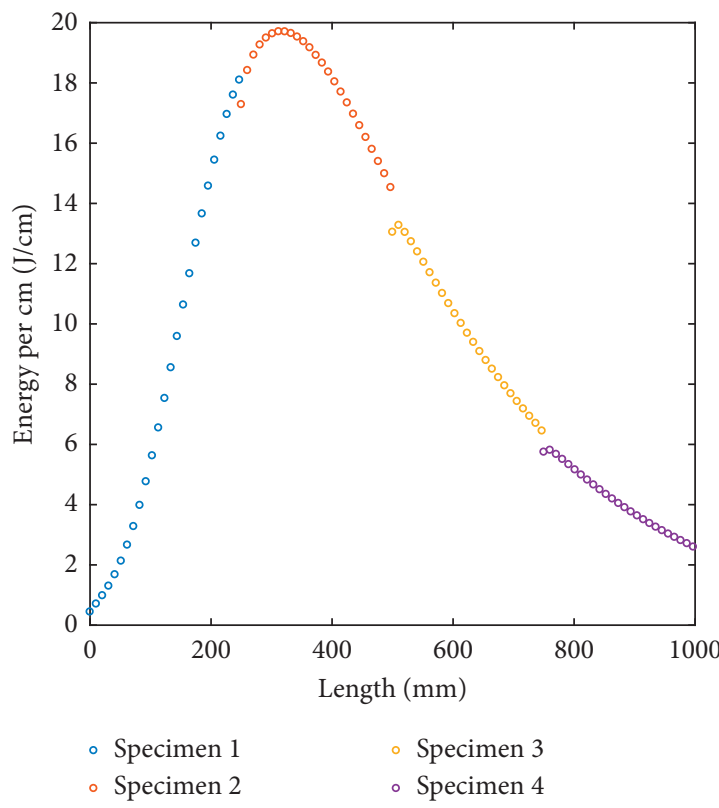

(a)

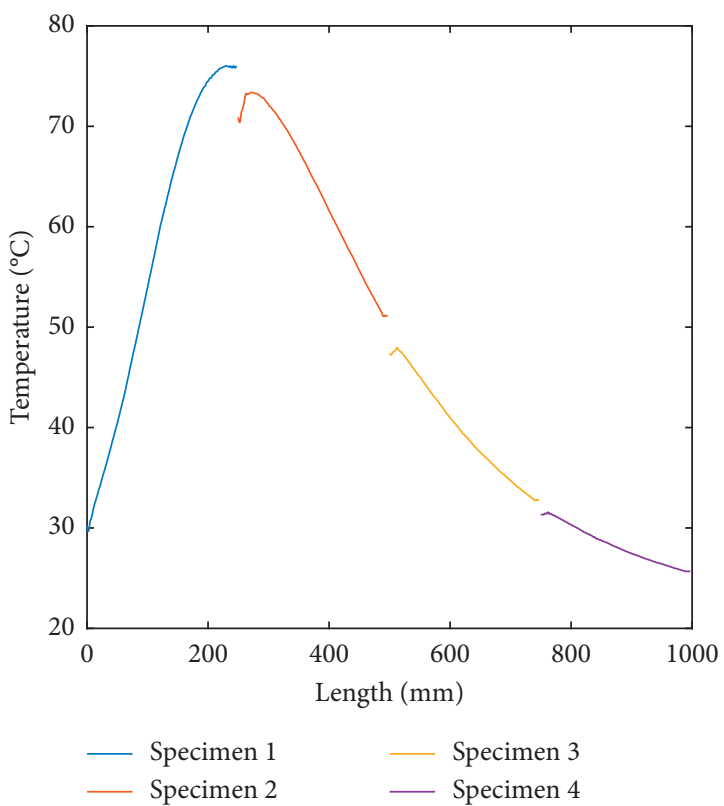

(c)

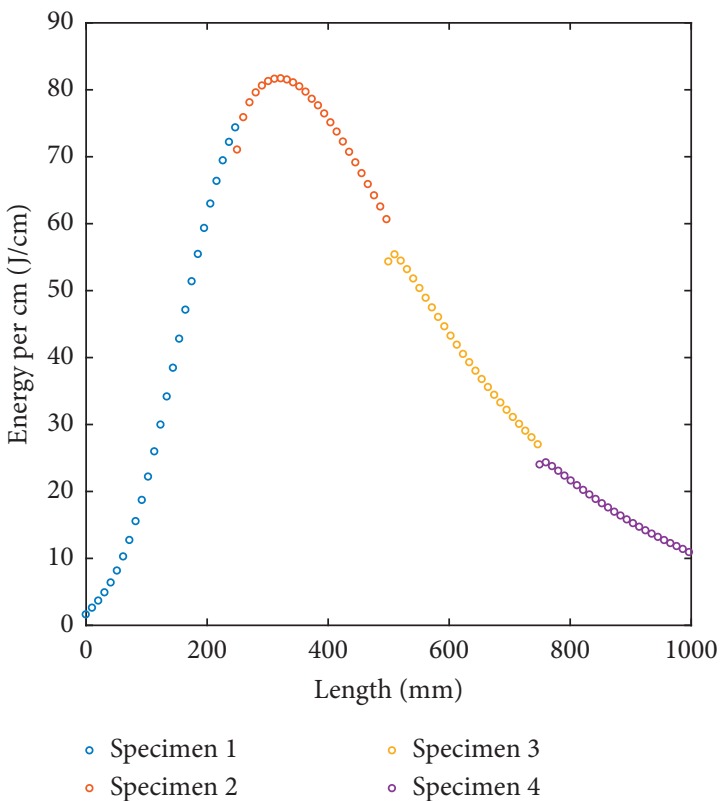

(b)

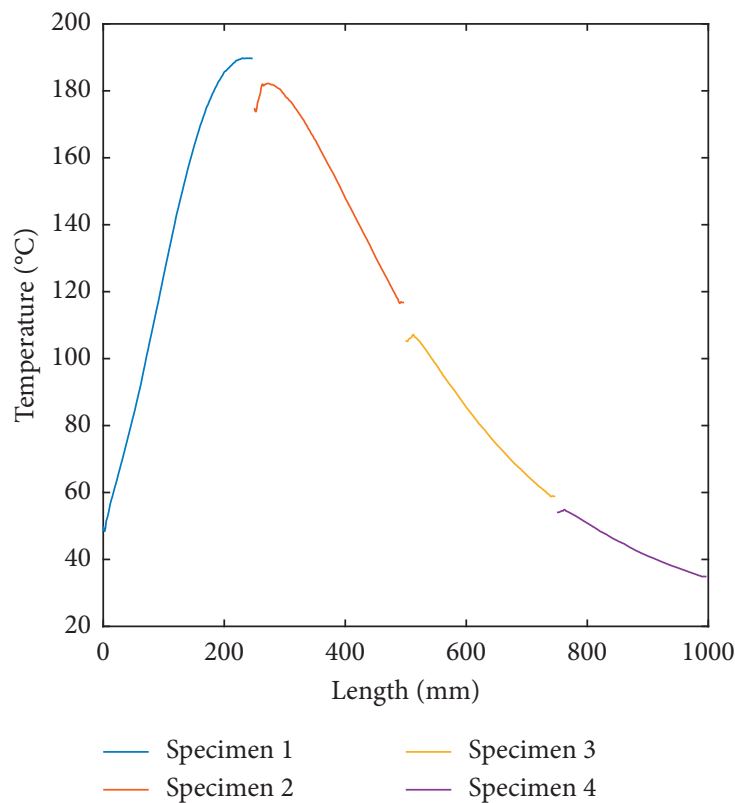

(d)

Figure 17: Energy deposited along the length of the specimens in the CuCD RHP target station for shots 128 (a) and 132 (b) and temperature along the length of specimens for each respective shot $(c, d)$.

The most loaded $\mathrm{CuCD}$ specimen can be seen to be the second in the target station-this specimen was chosen to model the dynamic phenomena in the structural analysis due to the lower signal-to-noise ratio achieved as a result of the higher deposited energy and subsequent larger amplitudes in the propagating waves. Figure 18 shows experimental measurements obtained from a thermal probe positioned on the outer surface of the second specimen at a length of $82.3 \mathrm{~mm}$, LF1 in Figure 14, compared with thermal analysis results for the modelled specimen, probed at the 


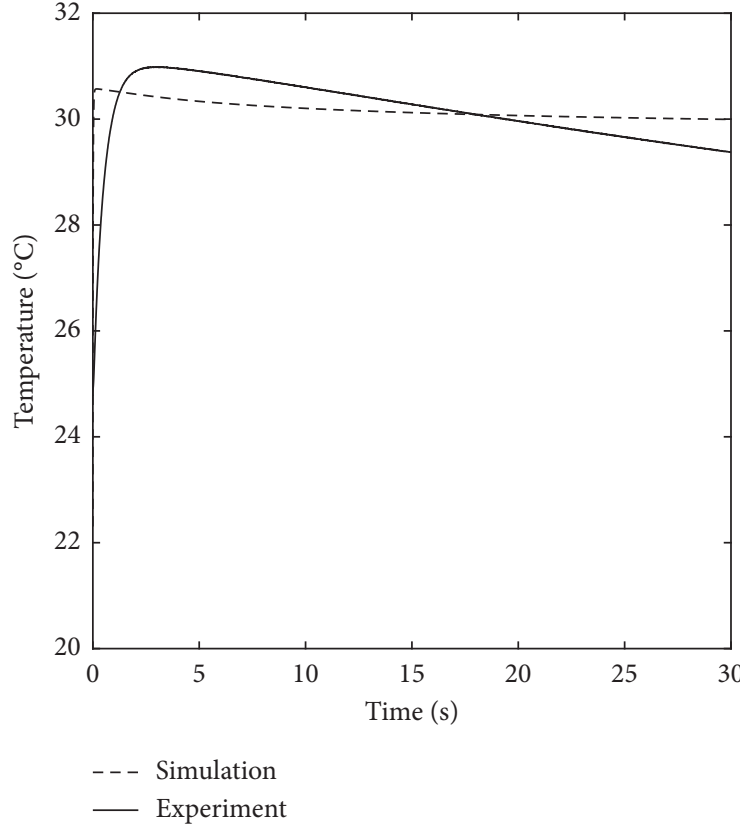

(a)

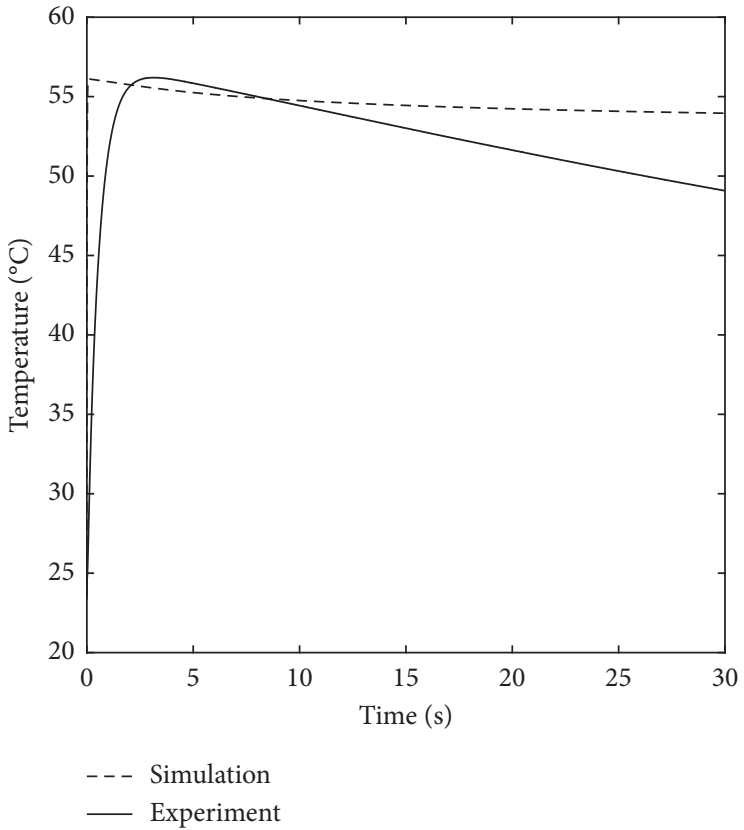

(b)

Figure 18: Thermal analysis results compared with experimental results for shot 128 and shot 132, probed on the surface of the second specimen at a length of $82.3 \mathrm{~mm}$.

same location. The thermal probe has a reaction time in the order of seconds and is not capable of capturing variations of temperature in the order of microseconds. This, coupled with issues related to contact resistance between the thermal probe and the tested specimens, results in a delay in experimental measurements to reach the maximum temperature when compared with the numerical analysis. The simulation can otherwise be seen to accurately predict the maximum temperature at the surface of the specimen.

The temperature field obtained from the thermal analyses was imported in a structural analysis modelling the material's dynamic behaviour upon beam impact. The dynamic longitudinal and flexural response was modelled by considering a simply supported specimen (modelling the experimental setup). Again, the second specimen in the target station (being the most loaded) was modelled, and results were probed at a length of $154 \mathrm{~mm}$ at a point at the centre of the specimen's bottom face. These results were compared with experimental results obtained from a longitudinal strain gauge attached to the specimen at the same position. The dynamic longitudinal response for shot 128 probed at a length of $154 \mathrm{~mm}$ on the bottom face of the specimen, obtained with the application of the material model described in the study, is compared with experimental results in Figure 19. Along with a minor but measurable difference in amplitude, the main discrepancy between the two signals can be seen to be in the longitudinal frequency, with the numerical result having a frequency of $11.8 \mathrm{kHz}$, compared with an experimental result of $13.0 \mathrm{kHz}$ (10\% difference). The speed of sound of the material-on which the frequency is dependent-is a function of the density of the specimen and the material's stiffness (Young's modulus in the elastic domain or $\delta \sigma / \delta \varepsilon$ in general).

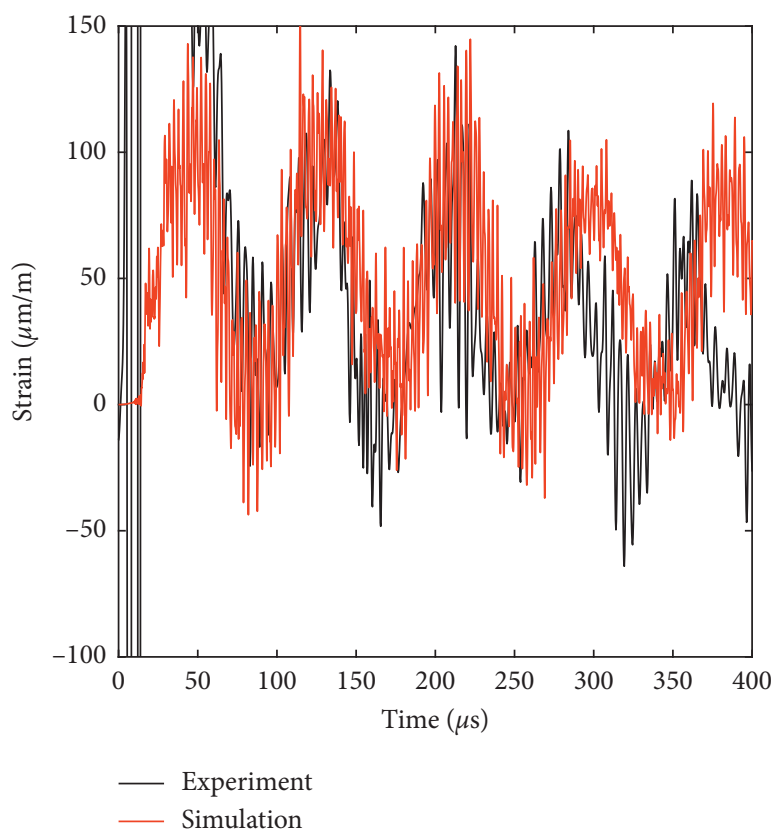

FIGURE 19: Longitudinal strain for shot 128, probed at a length of $154 \mathrm{~mm}$ on the bottom face of the second specimen. The numerical model with Young's modulus of $194 \mathrm{GPa}$ and the multilinear hardening model.

The relatively high variability inherent with this type of material (a result of the large amount of porosity) may cause a variation of properties between different specimen batches, e.g., between the ones tested in the characterisation campaign and those tested in the MultiMat experiment. The 
density measured in the characterisation campaign is assumed to be valid since this takes into account the material porosity suggesting that the variations are results of an underestimation of the material's stiffness in the model. The material model was thus updated with a higher Young's modulus of $220 \mathrm{GPa}$, a value that provides the best fit for longitudinal and flexural frequencies between experimental and numerical data, retaining all other properties. Simulation results compared with the experimental result for this scenario are shown in Figure 20. The increase in Young's modulus results in a 'compression' of the waveform (i.e., an increase in frequency), which leads to a better fit with experimental data, with an improved longitudinal frequency of $12.6 \mathrm{kHz}$. The benefits of using a multilinear hardening model, rather than an elastic model, can be clearly seen in Figure 21, which shows the result for a perfectly elastic (i.e., no plasticity and not multilinear) model with Young's modulus of $220 \mathrm{GPa}$. It can be seen that in this case the longitudinal frequency is retained to a certain extent, but the model fails to correctly simulate the high frequency content of the signal, which can be seen to have a much higher amplitude than the one observed in the experiment.

With regard to the flexural response, shown in Figure 22, for the same beam impact (shot 128), the numerical result (with multilinear hardening and a Young's Modulus of $220 \mathrm{GPa}$ ) can be seen to overestimate the amplitude of oscillation. This discrepancy is addressed by improving the material model with the introduction of Rayleigh damping to the analysis, with a Rayleigh damping ratio of $\zeta=8.61 \%$ at $450 \mathrm{~Hz}$ (the flexural frequency), for a stiffness coefficient $\beta=6.088 \times 10^{-5}$. The resulting waveform can be seen to closely follow the experimentally measured signal. With the implementation of Rayleigh damping at the flexural frequency, it can be also seen that information at higher frequencies is lost.

The implementation of the multilinear hardening model already produced a decay in amplitude of numerical results, as a result of dissipation, in the longitudinal response, as could be observed by comparing the numerical results in Figure 21 with those shown in Figure 20. Similarly, for the flexural response, the numerical results without the use of the Rayleigh damping coefficient (also shown in Figure 22) can also be seen to exhibit a reduction in amplitude in both the lower frequency flexural oscillations as well as in the higher frequency content. Therefore, a hardening model with a lower yield stress would induce an earlier plasticisation, which would allow for a degree of control of dissipation of the signal without requiring the need to resort to numerical damping.

A summary of the longitudinal and flexural frequencies obtained through the different models implemented is shown in Table 6.

The repeatability of the multilinear kinematic hardening model adopted was tested by simulating a higher intensity, central shot (pulse no. 132 in Table 5). The longitudinal responses, experimental and numerical, for shot 132, are shown in Figure 23. Similar to the previously modelled pulse, the simulation follows the experimental result with a good degree of accuracy in terms of wave shape, amplitude, and frequency for the longitudinal wave. The model can be seen to underestimate the amplitude of high frequency content in the signal-this can indicate that the hardening curve adopted is overstating the dissipation of energy at higher intensities. An additional factor, which needs to be considered when looking at this discrepancy, is the inhomogeneity of the material's microstructure, which is composed of diamond particles dispersed in a copper matrix. The diamond particle size is an important factor, especially when compared with the wavelength of the travelling wave since internal reflections in the material can become significant and have an effect on the high frequency content observed in the measured signal.

\section{Discussion}

The multilinear hardening material model obtained through the thermomechanical characterisation campaign described in this study proved accurate in replicating the shape of the experimentally obtained signal, improving on a purely elastic model in terms of longitudinal wave shape and amplitude relative to the experimentally obtained signal. Despite this, one can see that Young's modulus computed from the impulse excitation technique test measurements underestimates the value observed in the MultiMat experiment measurements. This is believed to be a result of the high variability of the material due to the high level of porosity present, as well as to possible errors in modelling the plastic part of the hardening curve.

Differences between the longitudinal wave results obtained experimentally and those generated numerically can be observed in terms of high frequency dissipative effects, as observed in Figure 23. This discrepancy suggests that the hardening model adopted does not encompass the full range of behaviour of the material, which is represented as a homogeneous entity in the numerical analyses conducted. On a mesoscale level, the material is actually composed of diamond particles dispersed in a copper matrix. This results in stress waves propagating through the material having to travel from one medium to another, which results in the wave splitting into respective transmitted and reflective portions in accordance with the shock impedance of the two constituents of the material. The longitudinal signal in experimental results can be seen to reduce in amplitude significantly following the first oscillation, which could similarly be related to this phenomenon. As mentioned, in this study, the effects mentioned were treated in a homogeneous model through the application of damping and the modification of the yield stress of the material, which results in a loss of energy and thus a dissipation of the propagating waves. This is fundamentally different from the wave-particle interaction discussed since in this case the mechanism in play is wave dispersion at the particle-matrix interface.

The following considerations are hence proposed for future study of the material:

\section{(i) The modelling of internal material effects}

Longitudinal strain signals measured experimentally can be seen to decay as the wave propagates through the material. This phenomenon is believed to be a result of the inhomogeneous mesoscale structure of $\mathrm{CuCD}$, which is composed of diamond particles (with particle size varying between 40 and $200 \mu \mathrm{m}$ ) in the copper matrix, resulting in the stress waves propagating through the medium being split 


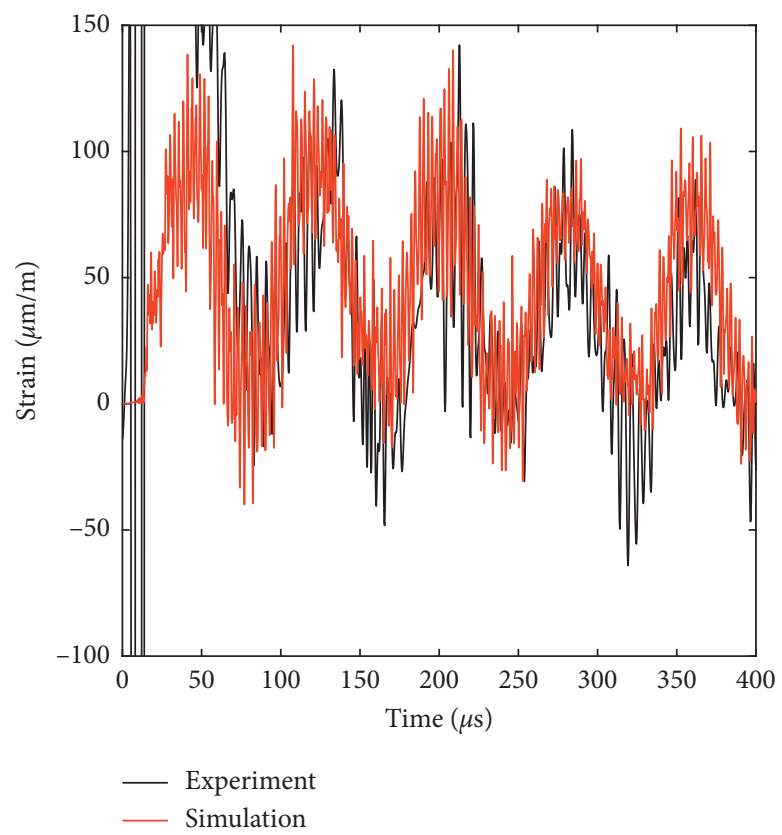

Figure 20: Longitudinal strain for shot 128, probed at a length of $154 \mathrm{~mm}$ on the bottom face of the second specimen. The numerical model with Young's modulus of $220 \mathrm{GPa}$ and the multilinear hardening model.

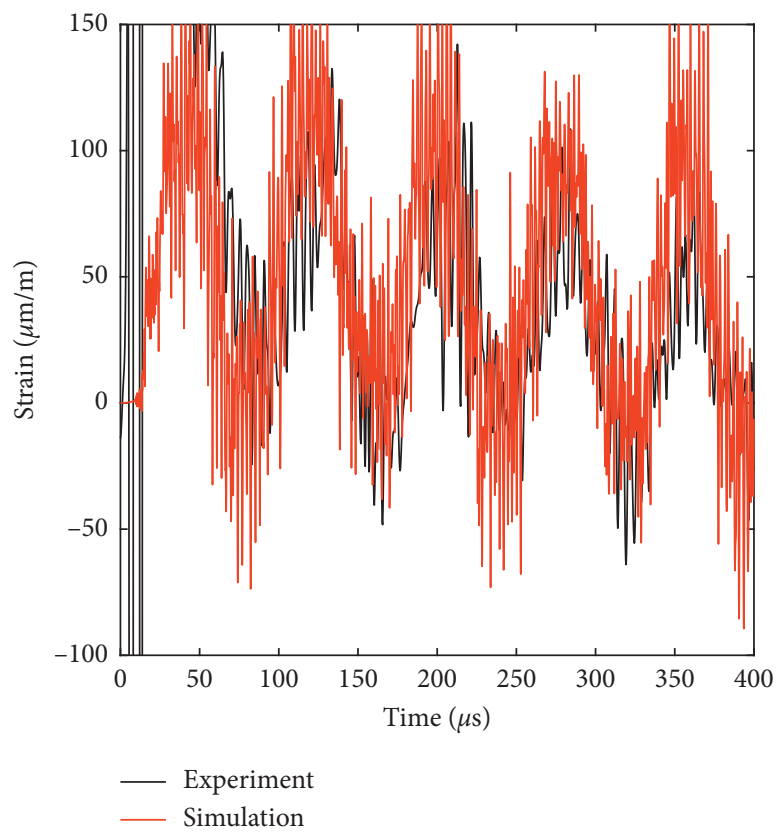

Figure 21: Longitudinal strain for shot 128 with a linear elastic model $(E=220 \mathrm{GPa})$, plotted against experimental data measured at a length of $154 \mathrm{~mm}$ on the bottom face of the second specimen.

into transmitted and reflected portions, depending on the shock impedance of the two materials, resulting in the perceived decay of the signal. While such a phenomenon conserves the total energy of the system, dissipative effects related to internal friction are also believed to be contributing to this decay. The effects related to the microstructure of the material can be studied by modelling the diamond particles on a mesoscale level, continuing on the work proposed by Carra [5]. Figure 24 shows a schematic of a homogeneous and a mesoscale model, the former assumes a material behaviour which is constant across the geometry, while the mesoscale model attempts to model the real-life scenario where, in the case of $\mathrm{CuCD}$, diamond particles are dispersed in a copper matrix.

(ii) Strain rate and temperature-dependent testing

The presented thermomechanical characterisation campaign (excluding the IET test) tested the material in quasi-static 


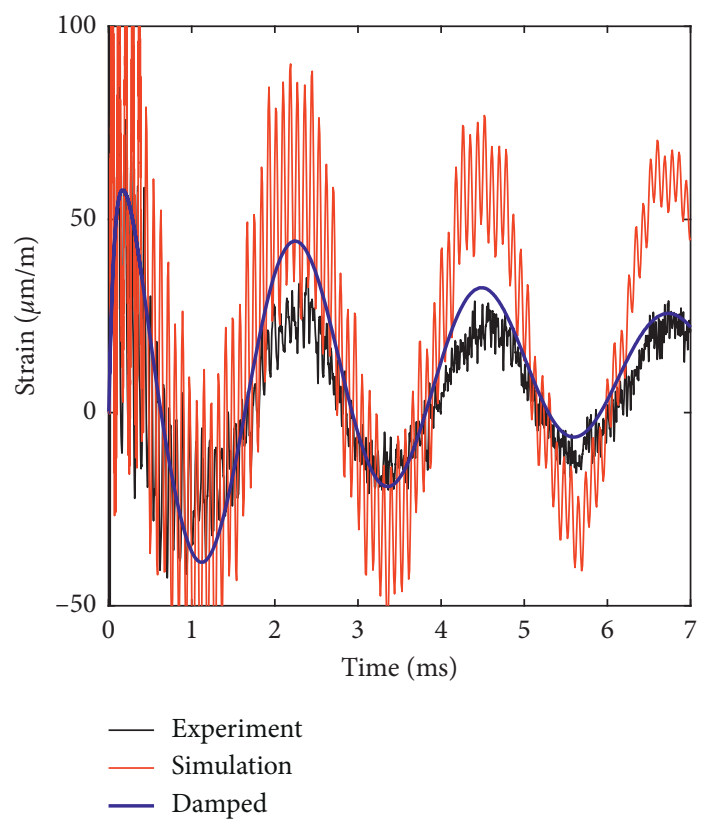

FIGURE 22: Flexural response for the multilinear model with $E=220 \mathrm{GPa}$ with and without Rayleigh damping for shot 128, probed at a length of $154 \mathrm{~mm}$ on the bottom face of the second specimen.

TABLE 6: Comparison of measured experimental frequencies with numerical results from perfectly elastic and multilinear hardening models.

\begin{tabular}{lcccr}
\hline & Experiment & $\begin{array}{c}\text { Simulation multilinear hardening } \\
\text { model } \\
E=194 \mathrm{GPa}\end{array}$ & $\begin{array}{c}\text { Simulation elastic } \\
\text { model } \\
E=220 \mathrm{GPa}\end{array}$ & $\begin{array}{c}\text { Simulation multilinear hardening } \\
\text { model } \\
E=220 \mathrm{GPa}\end{array}$ \\
\hline $\begin{array}{l}\text { Longitudinal frequency } \\
\text { (kHz) }\end{array}$ & $\mathbf{1 3 . 0 3}$ & 11.8 & 12.2 & $\mathbf{1 2 . 6}$ \\
Flexural frequency $(\mathrm{Hz})$ & $\mathbf{4 5 2}$ & 434 & 444 & $\mathbf{4 5 5}$ \\
\hline
\end{tabular}

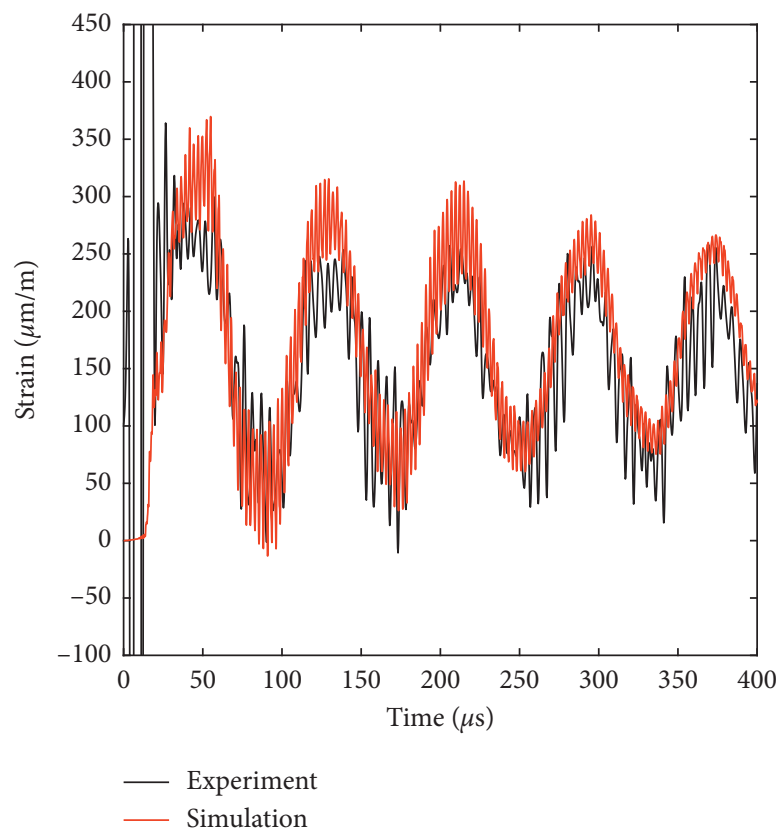

FIGURE 23: Longitudinal strain for shot 132, probed at a length of $154 \mathrm{~mm}$ on the bottom face of the second specimen. The numerical model with Young's modulus of $220 \mathrm{GPa}$ and the multilinear hardening model. 


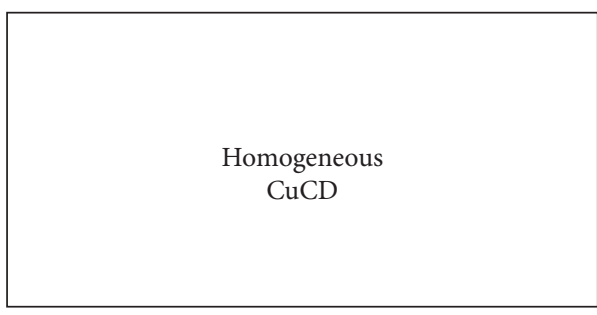

(a)

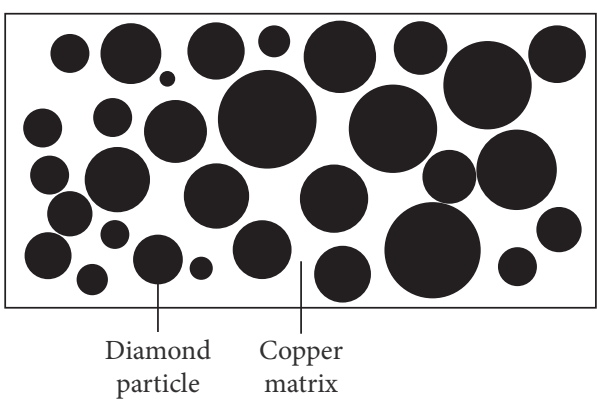

(b)

Figure 24: (a) Homogeneous and (b) mesoscale models.

conditions at room temperature, which can result in a difference in material behaviour compared with the dynamic conditions induced by sudden particle beam impacts. The splitHopkinson bar test is designed to characterise material behaviour at elevated strain rates and temperatures [41], allowing for the formulation of strength models, such as the Johnson-Cook and Zerilli-Armstrong models, which describe the material behaviour at extreme conditions. A split-Hopkinson bar test on CuCD RHP3434 samples will be conducted.

Additionally, future wave propagation problems and analyses could benefit from deviating from the finite element method. Alternatives based on machine learning exist, such as isogeometric analysis (IGA) and mesh-free methods. In such analyses, approximations for partial differential equation solutions can be found with an energy approach via the application of deep neural networks (DNNs) [42, 43]. An IGA approach provides some advantages with respect to FEA, namely, eliminating the geometric approximation error and reducing dissipation errors and numerical dispersion [44]. Finally, a sensitivity analysis quantifying the influence of all uncertain input parameters on the model's outputs would contribute in determining the impact of experimental uncertainty on results [45].

\section{Conclusions}

Copper diamond is a novel composite material currently being developed for possible implementation in the collimation system for use in future upgrades of the LHC and new particle accelerators, such as the high-luminosity LHC upgrade and the Future Circular Collider.

This study presents a material characterisation campaign for the material, conducted at CERN, mainly composed of measurements of temperature-dependent material properties, testing for the elastic properties of the material, and testing of the plastic behaviour of the material. The material model was then implemented in a simply-coupled thermomechanical analysis performed in ANSYS, the results of which were benchmarked with experimentally measured data from the MultiMat experiment conducted in CERN's HiRadMat facility. The homogeneous model implemented was able to replicate the longitudinal wave shape and amplitude, with some discrepancies observed in terms of the high frequency content in the longitudinal signal. This is believed to be a result of a combination of factors, namely, an overestimation of the plasticity induced in the material, as well as mesoscale effects related to the interaction of stress waves with diamond particles having a diameter which is close to the wavelength. With this in mind, a number of considerations for future study of the material are proposed, namely, the effect of the yield stress and plasticity on the dynamic material response, the modelling of internal material effects by considering the mesoscale structure of the material, as well as further testing of the material at high strain rates and temperatures.

\section{Data Availability}

The data used to support the findings of this study are available from the corresponding author upon request.

\section{Conflicts of Interest}

The authors declare that they have no conflicts of interest.

\section{Acknowledgments}

This project has received funding from the European Union's Horizon 2020 Research and Innovation Programme under grant no. 730871 .

\section{References}

[1] 'RHP-Technology GmbH', 2019, http://www.rhp-technology. $\mathrm{com} /$.

[2] J. Guardia-Valenzuela, "Development and characterisation of novel graphite-matrix composite material for thermal management applications," Escuela de Ingenieria Y Arquitectura, MSc thesis, Universidad de Zaragoza, Zaragoza, Spain, 2015.

[3] J. Guardia-Valenzuela, A. Bertarelli, F. Carra, N. Mariani, S. Bizzaro, and R. Arenal, "Development and properties of high thermal conductivity molybdenum carbide-graphite composites," Carbon, vol. 135, pp. 72-84, 2018.

[4] T. Weißgärber, Copper/Diamond Composites for Heat Sink Applications, https://www.nanoanalytik.fraunhofer.de/en/ library/CS1.html Fraunhofer IFAM, 2019. 
[5] F. Carra, Thermomechanical Response of Advanced Materials under Quasi Instantaneous Heating, PhD thesis, Politecnico di Torino, Italy, 2017.

[6] R. Bruce and S. Redaelli, CuCD-Based Tertiary Collimator, Presented at the HL-LHC TCC Meeting, CERN, Geneva, Switzerland, 2019.

[7] F. Carra, J. G. Valenzuela, P. Gradassi, and L. K. Mettler, Results on Simulations of New Materials and Composites, CERN, Geneva, Switzerlandhttps://edms.cern.ch/document/ $1325252 / 3$.

[8] A. Bertarelli, E. Berthome, V. Boccone et al., "An experiment to test advanced materials impacted by intense proton pulses at CERN HiRadMat facility," Nuclear Instruments and Methods in Physics Research Section B: Beam Interactions with Materials and Atoms, vol. 308, pp. 88-99, 2013.

[9] A. Bertarelli, High Energy Tests of Advanced Materials for Beam Intercepting Devices at CERN HiRadMat Facility, CERN, San Sebastián, Spain, 2012.

[10] I. Efthymiopoulos, HiRadMat: A New Irradiation Facility for Material Testing at CERN, CERN, San Sebastián, Spain, 2011.

[11] C. Torregrosa, "The Hiradmat 27 experiment: exploring highdensity materials response at extreme conditions for antiproton production," in Proceedings of the 7th International Particle Accelerator Conference (IPAC 2016), Busan, Korea, June 2016.

[12] F. Carra, A. Bertarelli, E. Berthomé, and C. Fichera, "'The "Multimat" experiment at CERN HiRadMat facility: advanced testing of novel materials and instrumentation for HL-LHC collimators," Journal of Physics: Conference Series, vol. 874, 2017.

[13] M. Pasquali, A. Bertarelli, C. Accettura et al., "Dynamic response of advanced materials impacted by particle beams: the MultiMat experiment," Journal of Dynamic Behaviour of Materials, vol. 5, pp. 266-295, 2019.

[14] A. Bertarelli, G. Arnau Izquierdo, F. Carra, A. Dallocchio, M. Gil Costa, and N. Mariani, "Research and development of novel advanced materials for next-generation collimators," in Proceedings of the 2nd International Particle Accelerator Conference, San Sebastian, Spain, 2011.

[15] M. Portelli, A. Bertarelli, F. Carra, M. Pasquali, N. Sammut, and P. Mollicone, "Numerical and experimental benchmarking of the dynamic response of $\mathrm{SiC}$ and TZM specimens in the MultiMat experiment," Mechanics of Materials, vol. 138, Article ID 103169, 2019.

[16] A. Bertarelli, F. Carra, M. Garlasche et al., "Innovative MoC-graphite composite for thermal management and thermal shock applications," in Proceedings of the 2015 31st Thermal Measurement, Modeling \& Management Symposium (SEMI-THERM), pp. 56-59, San Jose, CA, USA, 2015.

[17] 'DIL 402 Expedis Classic', https://www.netzsch-thermal-analysis. com/en/products-solutions/dilatometer/dil-402-expedis-classic/.

[18] ASTM International, Standard Test Method for Linear Thermal Expansion of Solid Materials with a Push-Rod Dilatometer, ASTM International, Standard ASTM E228-17, 2017.

[19] 'Sartorius Quintix Analytical Balance 224-1x', https://www. northernbalance.co.uk/product/sartorius-quintix-analyticalbalance-224-1x/.

[20] DIN, “Thermal analysis-differential thermal analysis (DTA) and differential scanning calorimetry (DSC)-general Principles," 2019. DIN, Standard DIN 51007:2019-04.

[21] J. Guardia-Valenzuela, Mathematical Model and Extrapolation for Thermal Diffusivity and Specific Heat, EDMS No. 1540215, 2015.
[22] T. D. Burchell, "Graphite: properties and characteristics," Comprehensive Nuclear Materials, no. 2, pp. 285-305, 2012.

[23] D. R. Lide, CRC Handbook of Chemistry and Physics, CRC Press, Boca Raton, FL, USA, 90th edition, 2009.

[24] M. Borg, A. Bertarelli, F. Carra et al., "Thermostructural characterization and structural elastic property optimization of novel high luminosity LHC collimation materials at CERN," Physical Review Accelerators and Beams, vol. 21, no. 3, 2018.

[25] ASTM International, 'Standard Test Method for Dynamic Young's Modulus, Shear Modulus, and Poisson's Ratio by Impulse Excitation of Vibration', ASTM International, Standard ASTM E1876-15, 2015.

[26] ASTM International, 'Standard Test Method for Dynamic Young's Modulus, Shear Modulus, and Poisson's Ratio for Advanced Ceramics by Impulse Excitation of Vibration', ASTM International, Standard ASTM C1259-15, 2015.

[27] ANSYS, Inc., ANSYS Mechanical User's Guide, Software Version: 15.0, ANSYS, Inc., Canonsburg, PA, USA, 2013.

[28] ASTM International, Standard Test Method for Flexural Strength of Manufactured Carbon and Graphite Articles Using Four-Point Loading at Room Temperature, ASTM International, Standard ASTM C651-15, 2015.

[29] L. Marrodan, M. P. Fiol, M. I. Gaitán et al., “Absence of latitudinal gradient in oligoclonal bands prevalence in Argentina," Multiple Sclerosis and Related Disorders, vol. 46, 2020102582.

[30] A. Bertarelli, "Permanent deformation of the LHC collimator jaws induced by shock beam impact: an analytical and numerical interpretation," in Proceedings of the European Particle Accelerator Conference (EPAC 2006), Edinburgh, UK, June 2006.

[31] M. Cauchi, O. Aberle, R. W. Assmann et al., "High energy beam impact tests on a LHC tertiary collimator at the CERN high-radiation to materials facility," Physical Review Special Topics-Accelerators and Beams, vol. 17, no. 2, Article ID 021004, 2014.

[32] E. Quaranta, Investigation of Collimator Materials for the High Luminosity Large Hadron Collider, Politecnico di Milano, Milano, Italy, 2017.

[33] A. Bertarelli, F. Carra, N. Mariani, and S. Bizzaro, Development and Testing of Novel Advanced Materials with Very High Thermal Shock Resistance, EuCARD-2 Scientific Report CERN-ACC-2014-0306, 2014, https://cds.cern.ch/record/ 1973365/files/CERN-ACC- 2014-0306.pdf.

[34] A. Bertarelli, Beam-Induced Damage Mechanisms and their Calculation, CERN, 0007-8328, 2016, https://e-publishing. cern.ch/index.php/CYR/article/view/234.

[35] M. Portelli, A. Bertarelli, F. Carra, L. K. Mettler, P. Mollicone, and N. Sammut, "Numerical simulation of long rods impacted by particle beams," Physical Review Accelerators and Beams, vol. 21, no. 6, 2018.

[36] A. Bertarelli, A. Dallocchio, and T. Kurtyka, "Dynamic response of rapidly heated cylindrical rods: longitudinal and flexural behavior," Journal of Applied Mechanics, vol. 75, no. 3, 2008.

[37] P. Sievers, "Elastic stress waves in matter due to rapid heating by an intense high-energy particle beam," CERN Technical Note No. LAB II/BT/74-2, 1974.

[38] H. Bargmann, "Dynamic response of external targets under thermal shock'," CERN Technical Note No. LAB II/BT/Int/ 73-3, 1973. 
[39] B. A. Boley, "Approximate analyses of thermally induced vibrations of beams and plates," Journal of Applied Mechanics, vol. 24, no. 3, pp. 413-416.

[40] A. Ferrari, P. R. Sala, A. Fassò, and J. Ranft, FLUKA: A MultiParticle Transport Code (Program Version 2005), CERN, Geneva, Switzerland, 2005.

[41] G. T. Gray, "Classic split-Hopkinson pressure bar testing," in ASM Handbook Volume 8: Mechanical Testing and Evaluation, Scientific Research Publishing Inc, Wuhan, China, 2000.

[42] E. Samaniego, C. Anitescu, S. Goswami et al., "An energy approach to the solution of partial differential equations in computational mechanics via machine learning: concepts, implementation and applications," Computer Methods in Applied Mechanics and Engineering, vol. 362, Article ID 112790, 2020.

[43] T. Rabczuk and T. Belytschko, "A three-dimensional large deformation meshfree method for arbitrary evolving cracks," Computer Methods in Applied Mechanics and Engineering, vol. 196, no. 29-30, pp. 2777-2799, 2007.

[44] L. Pegolotti, L. Dedè, and A. Quarteroni, "Isogeometric Analysis of the electrophysiology in the human heart: numerical simulation of the bidomain equations on the atria," Computer Methods in Applied Mechanics and Engineering, vol. 343, pp. 52-73, 2019.

[45] N. Vu-Bac, T. Lahmer, X. Zhuang, T. Nguyen-Thoi, and T. Rabczuk, "A software framework for probabilistic sensitivity analysis for computationally expensive models," Advances in Engineering Software, vol. 100, pp. 19-31, 2016. 